Article

\title{
UPLC-QTOF/MS-Based Nontargeted Metabolomic Analysis of Mountain- and Garden-Cultivated Ginseng of Different Ages in Northeast China
}

\author{
Hailin Zhu, Hongqiang Lin, Jing Tan, Cuizhu Wang, Han Wang, Fulin Wu, Qinghai Dong, \\ Yunhe Liu, Pingya Li * and Jinping Liu *D \\ Research Center of Natural Drugs, School of Pharmaceutical Sciences, Jilin University, Fujin Road 1266, \\ Changchun 130021, China; 13578965875@163.com (H.Z.); linhq17@mails.jlu.edu.cn (H.L.); \\ tanjing17@mails.jlu.edu.cn (J.T.); wangcz15@mails.jlu.edu.cn (C.W.); hanw17@mails.jlu.edu.cn (H.W.); \\ wufl17@mails.jlu.edu.cn (F.W.); dongqh17@mails.jlu.edu.cn (Q.D.); lyh133700@163.com (Y.L.) \\ * Correspondence: lipy@jlu.edu.cn (P.L.); liujp@jlu.edu.cn (J.L.); Tel.: +86-431-85619803 (P.L.)
}

Received: 3 December 2018; Accepted: 21 December 2018; Published: 21 December 2018

\begin{abstract}
Aiming at further systematically comparing the similarities and differences of the chemical components in ginseng of different ages, especially comparing the younger or the older and mountain-cultivated ginseng (MCG), 4, 5, 6-year-old cultivated ginseng (CG) and 12, 20-year-old MCG were chosen as the analytical samples in the present study. The combination of UPLC-QTOF-MS UNIFI platform and multivariate statistical analysis were developed to profile CGs and MCGs. By the screening analysis based on UNIFI, 126 chemical components with various structural types were characterized or tentatively identified from all the CG and MCG samples for the first time. The results showed that all the CG and MCG samples had the similar chemical composition, but there were significant differences in the contents of markers. By the metabolomic analysis based on multivariate statistical analysis, it was shown that $C_{4-6}$ years, $M_{C G}$ years and $M_{C G_{20}}$ years samples were obviously divided into three different groups, and a total of 17 potential age-dependent markers enabling differentiation among the three groups of samples were discovered. For differentiation from other two kinds of samples, there were four robust makers such as $\alpha$-linolenic acid, 9-octadecenoic acid, linoleic acid and panaxydol for $\mathrm{CG}_{4-6}$ years, five robust makers including ginsenoside $\operatorname{Re}_{1},-\mathrm{Re}_{2},-\mathrm{Rs}_{1}$, malonylginsenoside $R b_{2}$ and isomer of malonylginsenoside $R b_{1}$ for $M C_{20}$ years, and two robust makers, 24-hydroxyoleanolic acid and palmitoleic acid, for $\mathrm{MCG}_{12}$ years were discovered, respectively. The proposed approach could be applied to directly distinguish MCG root ages, which is an important criterion for evaluating the quality of MCG. The results will provide the data for the further study on the chemical constituents of MCG.
\end{abstract}

Keywords: mountain-cultivated ginseng; identification; metabolomic analysis; UPLC-QTOF-MS

\section{Introduction}

Ginseng, the king of herbs in the Orient, has always received a lot of attention, not only as a therapeutic medicinal herb, but also as a health supplement. According to the different growing environments and diverse cultivation methods, there two kinds of ginseng are distinguished in the Chinese Pharmacopoeia: cultivated ginseng (CG) and mountain-cultivated ginseng (MCG). CG is cultivated artificially in gardens, while MCG is grown for at least 10 years [1,2]. MCG, also called "Lin-Xia-Shan-Shen", can be regarded as a replacement of wild ginseng. MCG is of better quality than CG and offers more production than wild ginseng [3]. Actually, the adulteration or falsification of the cultivation age of MCG has always been a serious problem in the MCG commercial market. As we all know, the chemical components and biological activities of ginseng with different cultivation ages are 
distinct $[4,5]$, and more aged ginseng is usually of higher economic value. In an investigation of the characteristic components for distinguishing CG (4-7-year of age) and MCG (with 15-years of growth), 12 compounds, including ginsenoside $\mathrm{Ra}_{3}$ /isomer, gypenoside $\mathrm{XVII}$, quinquenoside $\mathrm{R}_{1}$, ginsenoside $\mathrm{Ra}_{7}$, notoginsenoside $\mathrm{Fe}$, ginsenoside $\mathrm{Ra}_{2}$, ginsenoside $\mathrm{Rs}_{6} / \mathrm{Rs}_{7}$, malonyl ginsenoside $\mathrm{Rc}$, malonyl ginsenoside $\mathrm{Rb}_{1}$, malonyl ginsenoside $\mathrm{Rb}_{2}$, palmitoleic acid, and ethyl linoleate were regarded as the characteristic chemical markers for the discrimination [6]. Recently, a UPLC/QTOF- MS-based metabolomics approach was applied to the global metabolite profiling of MCG leaf samples aged from 6 to 18 years, and the authors claimed that the approach could also be applied to discriminate MCG root ages indirectly [7]. It is undoubted that the developed method can be used as a standard protocol for discriminating and predicting MCG leaf ages directly, but there might be some inaccuracy and uncertainty when discriminating MCG root ages indirectly.

In the past decades, some analytical methods focusing on ginsenosides had been used to distinguish MCG from CG, such as thin layer chromatography (TLC), or high performance liquid chromatography (HPLC) [8,9]. However, these technologies require lots of time and energy, and the results cannot provide a comprehensive or accurate discrimination between them. Currently, untargeted metabolomics, combined with multivariate statistical methods such as principal component analysis (PCA) and orthogonal partial least squares discriminant analysis (OPLS-DA), are widely used to profile diverse classes of metabolites and to better understand the chemical diversity and the multiple pharmacological effects of ginsenosides or ginseng [10,11]. Given the multi-component property, the combination of LC-MS-based metabolomic profiling with multivariate statistical analysis methods was used as a rapid means of characterization and was increasingly applied for analyzing ginseng from different herbs, cultivation environments/areas, cultivation ages or different parts [12,13]. As an example, for different herbs belonging to the same genus, specific biomarkers including chikusetsusaponin IVa, ginsenoside Rf and ginsenoside Rc were selected and verified for ginseng [14]. In another example of different parts analysis, the metabolic profiles of root, leaf, flower bud, berry and seed of ginseng were investigated [12,15]. In addition, the approach for the discrimination of different red ginseng root parts was reported. As a result, fine roots had the highest protopanaxadiol (PPD)/protopanaxatriol (PPT) ratio, which could clearly distinguish the main roots from the lateral roots and fine roots parts [16]. Such analysis was also applied to make metabolite profiling and age discrimination of 4- and 6-year- old red ginseng [17], or 1-6 years ginseng [18].

In addition, UNIFI, the automated data processing software, is an integrated informatics platform that possesses the ability to incorporate scientific library into a streamlined workflow, aiming at identifying chemical components from complex raw data [19]. The combination of UPLC separation, $\mathrm{Q} / \mathrm{TOF}-\mathrm{MS}$ detection and UNIFI platform has been frequently applied in the characterization of chemical constituents of herbs [20,21].

Normally, CG is harvested after a 4-6 years cultivation period, and MCG is collected at ages of 10-20 years. To develop a more direct and more efficient discrimination method for the cultivation ages and to explore potential age-dependent markers, we chose 4, 5, 6-year-old CG and 12, 20-year-old MCG as the analytical samples in the present study. UPLC-QTOF-MSE, UNIFI platform and multivariate statistical analysis were then used to profile these two kinds of ginseng. The aims were to systematically screen the chemical components and to perform the non-targeted metabolomic analysis, and in turn will lay the foundation for the establishment of CG and MCG quality criteria in the future. In one hand, this study will reveal the structural diversity of secondary metabolites and the different patterns in CG and MCG. In the other hand, the present study could provide a reference point for a reliable, accurate method for distinguishing among CG and MCG samples of different ages. 


\section{Materials and Methods}

\subsection{Materials and Reagents}

A total of 40 batches of CG and MCG root products, including 24 batches of CGs and 16 batches of MCGs, were collected from different cultivation areas in Jilin Province, the main source of ginseng in China. A detailed sample list is given in Table 1. All samples were harvested and collected by Professor Li Ping-ya from Jilin University Institute of Frontier Medical Science, according to China Pharmacopoeia (2015 version) [22]. Voucher specimens have been deposited at the Research Center of Nature Drug, School of Pharmaceutical Sciences, Jilin University, Changchun, China.

Table 1. Details of the MCG and CG samples.

\begin{tabular}{|c|c|c|}
\hline Sample No. & Source & Collection Time \\
\hline $\begin{array}{c}\mathrm{CG}_{3 \text { years }}-1, \mathrm{CG}_{3 \text { years }}-2 ; \mathrm{CG}_{4 \text { years }}-1, \mathrm{CG}_{4 \text { years }}-2 ; \\
\mathrm{CG}_{5 \text { years }}-1, \mathrm{CG}_{5 \text { years }}-2 ; \mathrm{MCG}_{12 \text { years }}-1, \\
\mathrm{MCG}_{12 \text { years }}-2 ; \mathrm{MCG}_{20 \text { years }}-1, \mathrm{MCG}_{2 \text { years }}-2\end{array}$ & $\begin{array}{l}\text { Ji' an City, Jilin Province, } \\
\text { China }\end{array}$ & 2017.09-2017.10 \\
\hline $\begin{array}{c}\mathrm{CG}_{3 \text { years }}-3, \mathrm{CG}_{3 \text { years }}-4 ; \mathrm{CG}_{4 \text { years }}-3, \mathrm{CG}_{4 \text { years }}-4 ; \\
\mathrm{CG}_{5 \text { years }}-3, \mathrm{CG}_{5 \text { years }}-4 ; \mathrm{MCG}_{12 \text { years }}-3 \\
\mathrm{MCG}_{12 \text { years }}-4 ; \mathrm{MCG}_{20 \text { years }}-3, \mathrm{MCG}_{20 \text { years }}-4\end{array}$ & $\begin{array}{l}\text { Fusong County, Jilin } \\
\text { Province, China }\end{array}$ & 2017.09-2017.10 \\
\hline $\begin{array}{c}\mathrm{CG}_{3 \text { years }}-5, \mathrm{CG}_{3 \text { years }}-6 ; \mathrm{CG}_{4 \text { years }}-5, \mathrm{CG}_{4 \text { years }}-6 \\
\mathrm{CG}_{5 \text { years }}-5, \mathrm{CG}_{5 \text { years }}-6 ; \mathrm{MCG}_{12 \text { years }}-5 \\
\mathrm{MCG}_{12 \text { years }}-6 ; \mathrm{MCG}_{20 \text { years }}-5, \mathrm{MCG}_{20 \text { years }}-6\end{array}$ & $\begin{array}{l}\text { Tonghua City, Jilin } \\
\text { Province, China }\end{array}$ & 2017.09-2017.10 \\
\hline $\begin{array}{c}\mathrm{CG}_{3 \text { years }}-7, \mathrm{CG}_{3 \text { years }}-8 ; \mathrm{CG}_{4 \text { years }}-7, \mathrm{CG}_{4 \text { yeass }}-8 ; \\
\mathrm{CG}_{5 \text { years }}-7, \mathrm{CG}_{5 \text { years }}-8 ; \mathrm{MCG}_{12 \text { years }}-7, \\
\mathrm{MCG}_{12 \text { years }}-8 ; \mathrm{MCG}_{20 \text { years }}-7, \mathrm{MCG}_{20 \text { years }}-8\end{array}$ & $\begin{array}{l}\text { Jingyu Country, Jilin } \\
\text { Province, China }\end{array}$ & 2017.09-2017.10 \\
\hline
\end{tabular}

Acetonitrile, methanol were all UPLC-MS pure grade (Fisher Scientific Inc., Geel, Belgium). Formic acid (MS grade) was purchased from Sigma-Aldrich (St. Louis, MO, USA). Leucine enkephaline was provided by Waters (Waters Technologies, Milford, MA., USA). Distilled water was prepared in-house via a Millipore water purification system (Millipore, Billerica, MA, USA). All other chemicals were analytical grade. For reference substances, ginsenoside $F_{1}$ (R20151040), - $F_{2}$ (R20151040), notoginsenoside $R_{1}$ (R20170210), notoginseno- side $R_{4}$ (R20170212) were provided by the Research Center of Natural Drugs, School of Pharmaceutical Sciences, Jilin University, China. Ginsenoside $\mathrm{Rb}_{1},-\mathrm{Rb}_{2},-\mathrm{Rb}_{3},-\mathrm{Rc},-\mathrm{Rd},-\mathrm{Re},-\mathrm{Rf},-\mathrm{F}_{5},-\mathrm{Rg}_{1}, 20(R)-\mathrm{Rg}_{2}, 20(S)-\mathrm{Rh}_{1}, 20(R)-\mathrm{Rh}_{1}, 20(S)-\mathrm{Rg}_{3}, 20(R)-\mathrm{Rg}_{3}$, -Ro, gypenoside XVII, ginsenoside $\mathrm{Rs}_{1},-\mathrm{Rs}_{2}$ were isolated in our laboratory and identified by spectroscopic data. Adenine (101774299), tryptophane (73-22-2), palmitoleic acid (101491588) were purchased from Sigma-Aldrich. Notoginsenoside Fe (8105-29-5), D-adenosine (110879- 200502), histidine (624-200304) were purchased from the National Institutes for Food and Drug Control. Ginsenoside $\operatorname{Rg}_{5}$ (wkq16051002, Victory Biological Technology Co., Ltd., Sichuan, China), $\alpha$-linoleic acid (B21469; Yuanye Biological Technology Co., Ltd., Shanghai, China), D-arginin (130701; Nuoye Biological Engineering Co., Ltd., Anhui, China) and phenylpropionic acid (A20160211), quillaic acid (A20171109) were purchased from Beijing Zhongke Quality Inspection Biotechnology Co., Ltd. (Beijing, China) with the Chinese National Standard Sieve No. 3 (R40/3 series).

\subsection{Sample Preparation and Extraction}

All the CG and MCG samples were air-dried, grinded (Baijie Stainless Steel Grinder, BJ-800A, Deqing Baijie Electric Apllicance Co. Ltd., Zhejiang, China) and sieved (Chinese National Standard Sieve No. 3, R40/3 series) to get the homogeneous powder respectively. Then, the powder of 40 samples (200 mg accurately weighed per sample) were refluxed respectively with $85 \%$ methanol (2 L) at $80{ }^{\circ} \mathrm{C}$ for three times $(2 \mathrm{~h}, 2 \mathrm{~h}, 1 \mathrm{~h}$ each time, respectively). Then, the extracts of each sample were combined, concentrated and evaporated to dryness. Each powder was dissolved in $5.0 \mathrm{~mL}$ of $80 \%$ methonal. After being filtered, each methanolic solution was injected directly into UPLC system. 
Meanwhile, $20 \mu \mathrm{L}$ aliquots of each CG and MCG sample were mixed to obtain a quality control (QC) sample, which contained all of the components in the analysis. The QC sample was run randomly to monitor the stability of the system. All of the above solutions were stored at $4{ }^{\circ} \mathrm{C}$ prior to LC-MS analysis and the injection volume was $2 \mu \mathrm{L}$.

\subsection{UPLC/QTOF-MSE}

The chromatographic separation and mass spectrometry detection were conducted on the Waters Acquity UPLC system coupled with a Xevo G2-S QTOF mass spectrometer equipped with an electrospray ionization source (ESI). Separation was performed on Waters ACQUITY UPLC BEH $\mathrm{C}_{18}$ column $(100 \mathrm{~mm} \times 2.1 \mathrm{~mm}, 1.7 \mu \mathrm{m})$ at $40{ }^{\circ} \mathrm{C}$. The mobile phase consisted of eluent $\mathrm{A}(0.1 \%$ formic acid aqueous solution) and eluent $\mathrm{B}(0.1 \%$ formic acid in acetonitrile) at flow rate of $0.4 \mathrm{~mL} / \mathrm{min}$ with the following gradient program: 0 2 $\mathrm{min}, 10 \%$ (B); $2 \sim 26 \mathrm{~min}, 10 \% \sim 100 \%$ (B); $26 \sim 28 \mathrm{~min}, 100 \%$ (B); 28 28.1 $\mathrm{min}, 100 \%$ 10\% (B); 28.1 30 $\mathrm{min}, 10 \%$ (B). Mixtures of 10/90 and 90/10 water/acetonitrile were the strong wash and the weak wash solvent, respectively. The optimized conditions were employed: source temperature was $120^{\circ} \mathrm{C}$, the desolvation temperature was $300{ }^{\circ} \mathrm{C}$, capillary voltage was $2.6 \mathrm{kV}\left(\mathrm{ESI}^{+}\right)$or $2.2 \mathrm{kV}\left(\mathrm{ESI}^{-}\right)$, cone voltage was $40 \mathrm{~V}$, desolvation gas flow was $800.0 \mathrm{~L} / \mathrm{h}$, cone gas flow was $50 \mathrm{~L} / \mathrm{h}$. The energy of low energy function and the collision energy of high energy function were set at $6 \mathrm{~V}$ and $20 \mathrm{~V} \sim 40 \mathrm{~V}$ respectively in $\mathrm{MS}^{\mathrm{E}}$ mode. The mass spectrometer was calibrated with sodium formate in the range of 200-1500 Da. The lockmass compound used was leucine- enkephaline (external reference to the ion $m / z 556.2771$ in positive mode and 554.2615 in negative mode). Data were collected with Masslynx ${ }^{\mathrm{TM}}$ V4.1 workstation in continuum mode.

\subsection{Chemical Information Database for the Components of CG and MCG}

In addition to the Waters Traditional Medicine Library in UNIFI software, a systematic investigation of chemical constituents from the target herbs based on the literature was conducted. A self-built database of compounds, such as saponins, flavonoids, volatile oil, amino acids and so on, isolated from CG and MCG was established by searching online databases such as China Journals of Full-Text Database (CNKI), PubMed, Medicine, Web of Science and ChemSpider. The name, molecular formula and structure of components from CG and MCG were obtained in the database.

\subsection{The Screening Analysis Based on UNIFI Platform}

UNIFI 1.7.0 software (Waters, Manchester, UK) was used to perform the screening analysis on the structural characteristics and MS fragmentation behaviors, especially for characteristic fragments. Main parameters were set as follows: peak intensity of high energy over 200 counts and the peak intensity of low energy over 1000 counts were the selected parameters in peak detection; mass error up to $\pm 10 \mathrm{ppm}$ for identified compound; retention time tolerance was set in the range of $\pm 0.1 \mathrm{~min}$; positive adducts containing $+\mathrm{H},+\mathrm{Na}$ or negative adducts containing $-\mathrm{H},+\mathrm{HCOOH}$ were all selected; the reference compound was leucine-enkephalin ( 556.2766 for positive ion, 554.2620 for negative ion). The MS raw data were processed using the streamlined workflow of UNIFI software to quickly identify the chemical components that met the match criteria with the in-house Traditional Medicine Library and the self-built database [20,21].

\subsection{The Metabolomics Analysis Based on Multivariate Statistical Analysis}

To differentiate MCG and CG, MarkerLynx XS V4.1 software (Waters, Milford, DE, USA) was used to process the raw data by deconvolution, alignment, data reduction and to perform the multivariate statistical analysis [20,21]. The following steps were performed: acquiring data, creating a MarkerLynx processing method, processing the acquired data and viewing results Extended Statistics (XS) Viewer. The main parameters in the method set to process the raw data were as follows: retention time range 5-28 min, mass range 200-1400 Da, mass tolerance $5 \mathrm{mDa}$, intensity threshold 2000 counts, mass 
window $0.05 \mathrm{Da}$, retention time window $0.20 \mathrm{~min}$. In resulting database list, RT- $m / z$ pairs represent an identifier of ion in the order of their elution time. The same value of RT and $m / z$ in different batches of samples were regarded as the same compound. Multivariate statistical analysis was then performed to find the potential biomarkers that significantly contributed to the difference among the groups. During the analysis, principal component analysis (PCA) was firstly used to show the maximum variation and pattern recognition in order to get the overview and classification, and the orthogonal projections to latent structures discriminant analysis (OPLS-DA) was then performed aiming to get the maximum separation between two groups. S-plots was then available to provide visualization of the OPLS-DA predictive component loading to facilitate model interpretation. Variable importance for the projection (VIP) was also used to help screen the different components, and the metabolites with VIP value above 1.0 were considered as potential markers. Additionally, a permutation test was performed to provide reference distributions of the $\mathrm{R}^{2} / \mathrm{Q}^{2}$-values that could indicate the statistical significance. Simca 15.0 software (Umetrics, Malmö, Sweden) was used to show the analysis results.

\section{Results and Discussion}

\subsection{Identification of Components from MCG and CG Based on UNIFI Platform}

As a result of our analysis, a total of 126 compounds, including triterpenoids (the main ingredients), flavonoids, organic acids and organic acid esters, alcohol phenols, aldehyde ketones and amino acids, etc., were characterized or tentatively identified from the MCG and CG in both ESI ${ }^{+}$and $\mathrm{ESI}^{-}$modes. 85 compounds were identified in $\mathrm{ESI}^{+}$mode and 41 compounds were identified in $\mathrm{ESI}^{-}$ mode. Base peak intensity (BPI) chromatograms are shown in Figure 1, the identification information is listed in Table 2, and the chemical structures are shown in Figure 2. 

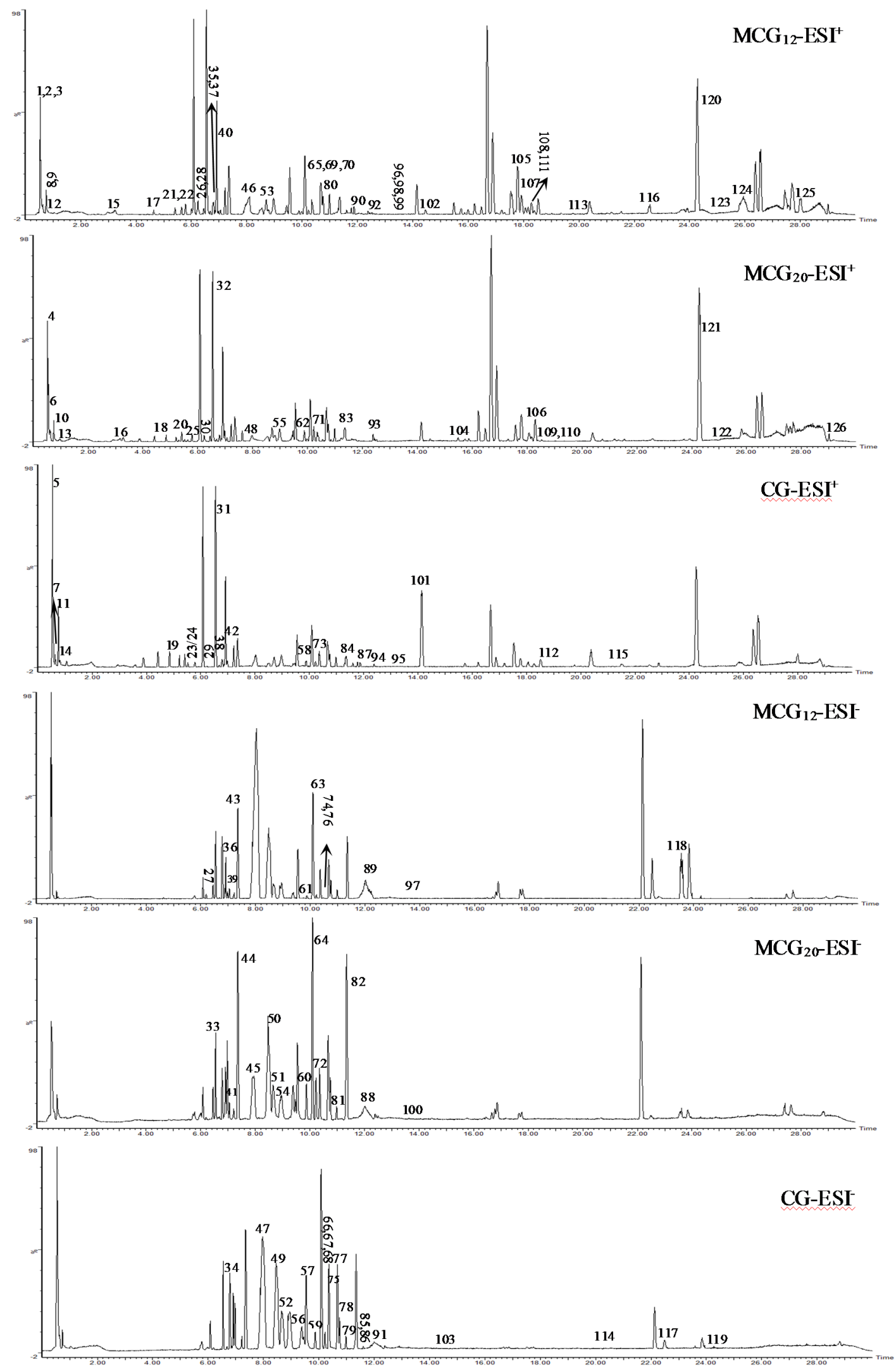

Figure 1. The representative BPI chromatograms of CG and MCG in positive and negative modes. 
Table 2. Compounds identified from MCG and CG by UPLC-QTOF-MSE

\begin{tabular}{|c|c|c|c|c|c|c|c|c|c|}
\hline No. & $t_{R}(\min )$ & Formula & $\begin{array}{l}\text { Calculated } \\
\text { Mass (Da) }\end{array}$ & $\begin{array}{l}\text { Theoretical } \\
\text { Mass (Da) }\end{array}$ & $\begin{array}{l}\text { Mass Error } \\
\quad(\mathrm{ppm})\end{array}$ & MS $^{\mathrm{E}}$ Fragmentation & Identification & Sources & Ref \\
\hline 1 & 0.49 & $\mathrm{C}_{5} \mathrm{H}_{8} \mathrm{~N}_{2} \mathrm{O}_{5}$ & 176.0431 & 176.0433 & -1.5 & $\begin{array}{l}177.0503[\mathrm{M}+\mathrm{H}]^{+} ; 130.0495\left[\mathrm{M}-2 \times \mathrm{OH}-\mathrm{NH}_{2}\right]^{+} \\
175.1188\left[\mathrm{M}+\mathrm{H}^{+} ; 158.0912\left[\mathrm{M}-\mathrm{NH}_{2}\right]^{+}\right.\end{array}$ & Dencichine & $\mathrm{CG}, \mathrm{MCG}_{12}, \mathrm{MCG}_{20}$ & [23] \\
\hline 2 & 0.54 & $\mathrm{C}_{6} \mathrm{H}_{14} \mathrm{~N}_{4} \mathrm{O}_{2}$ & 174.1115 & 174.1117 & -1.1 & $\begin{array}{l}116.0704\left[\mathrm{M}-\mathrm{NH}_{2}-\mathrm{CN}_{2} \mathrm{H}_{2}\right]^{+} ; 114.1015\left[\mathrm{M}-\mathrm{NH}_{2}\right. \\
\left.-\mathrm{CHO}_{2}\right]^{+}\end{array}$ & Adipodihydrazide & $\mathrm{CG}, \mathrm{MCG}_{12}, \mathrm{MCG}_{20}$ & a \\
\hline 3 & 0.55 & $\mathrm{C}_{30} \mathrm{H}_{22} \mathrm{O}_{10}$ & 542.1257 & 542.1213 & 8.1 & $\begin{array}{l}543.1330[\mathrm{M}+\mathrm{H}]^{+} ; 273.0833\left[\mathrm{M}-\mathrm{C}_{15} \mathrm{H}_{10} \mathrm{O}_{5}\right]^{+} ; \\
242.1025\left[\mathrm{M}-\mathrm{OH}-\mathrm{C}_{15} \mathrm{H}_{10} \mathrm{O}_{6}\right]^{+} ; 127.0388[\mathrm{M}- \\
\left.\mathrm{C}_{24} \mathrm{H}_{17} \mathrm{O}_{7}\right]^{+} ; 116.0703\left[\mathrm{M}-2 \times \mathrm{OH}-\mathrm{C}_{21} \mathrm{H}_{12} \mathrm{O}_{8}\right]^{+} ; \\
109.0284\left[\mathrm{M}-\mathrm{C}_{24} \mathrm{H}_{15} \mathrm{O}_{8}\right]^{+}\end{array}$ & Chamaejasmine & $\mathrm{CG}, \mathrm{MCG}_{12}, \mathrm{MCG}_{20}$ & a \\
\hline 4 & 0.59 & $\mathrm{C}_{12} \mathrm{H}_{22} \mathrm{O}_{11}$ & 342.1156 & 342.1162 & -1.6 & $\begin{array}{l}\text { 365.1055[M + Na }]^{+} ; 203.0550\left[\mathrm{M}-\mathrm{OH}-\mathrm{C}_{4} \mathrm{H}_{8} \mathrm{O}_{4}\right]^{+} \\
185.0444\left[\mathrm{M}-2 \times \mathrm{OH}-\mathrm{C}_{4} \mathrm{H}_{8} \mathrm{O}_{4}\right]^{+}\end{array}$ & $\alpha$-Maltose & $\mathrm{CG}, \mathrm{MCG}_{12}, \mathrm{MCG}_{20}$ & [24] \\
\hline 5 & 0.69 & $\mathrm{C}_{6} \mathrm{H}_{14} \mathrm{~N}_{4} \mathrm{O}_{2}$ & 174.1115 & 174.1117 & -0.9 & $175.1188[\mathrm{M}+\mathrm{H}]^{+}$ & D-Arginin & $\mathrm{CG}, \mathrm{MCG}_{12}, \mathrm{MCG}_{20}$ & $\mathrm{~s}$ \\
\hline 6 & 0.74 & $\mathrm{C}_{19} \mathrm{H}_{18} \mathrm{O}_{11}$ & 422.0842 & 422.0849 & -1.7 & $\begin{array}{l}423.0915[\mathrm{M}+\mathrm{H}]^{+} ; 268.1040\left[\mathrm{M}+\mathrm{H}-\mathrm{C}_{6} \mathrm{H}_{6} \mathrm{O}_{5}\right]^{+} ; \\
119.0349\left[\mathrm{M}-\mathrm{C}_{15} \mathrm{H}_{10} \mathrm{O}_{7}\right]^{+}\end{array}$ & Isomangiferin & $\mathrm{CG}, \mathrm{MCG}_{12}, \mathrm{MCG}_{20}$ & [25] \\
\hline 7 & 0.75 & $\mathrm{C}_{5} \mathrm{H}_{5} \mathrm{~N}_{5}$ & 135.0546 & 135.0545 & 0.5 & $136.0618[\mathrm{M}+\mathrm{H}]^{+} ; 119.0352\left[\mathrm{M}-\mathrm{NH}_{2}\right]^{+}$ & Adenine & $\mathrm{CG}, \mathrm{MCG}_{12}, \mathrm{MCG}_{20}$ & $\mathrm{~s}$ \\
\hline 8 & 0.76 & $\mathrm{C}_{10} \mathrm{H}_{13} \mathrm{~N}_{5} \mathrm{O}_{4}$ & 267.0974 & 267.0968 & 2.4 & $\begin{array}{l}268.1050\left[\mathrm{M}+\mathrm{H}^{+} ; 136.0618\left[\mathrm{M}-\mathrm{C}_{5} \mathrm{H}_{9} \mathrm{O}_{4}\right]^{+}\right. \\
119.0352\left[\mathrm{M}-\mathrm{C}_{5} \mathrm{H}_{9} \mathrm{O}_{4}-\mathrm{NH}_{2}\right]^{+}\end{array}$ & D-Adenosine & $\mathrm{CG}, \mathrm{MCG}_{12}, \mathrm{MCG}_{20}$ & $\mathrm{~s}$ \\
\hline 9 & 0.80 & $\mathrm{C}_{20} \mathrm{H}_{15} \mathrm{NO}_{6}$ & 365.0876 & 365.0899 & -6.3 & $\begin{array}{l}366.0949[\mathrm{M}+\mathrm{H}]^{+} \\
561.1569[\mathrm{M}+\mathrm{Na}]^{+} ; 393.1138\left[\mathrm{M}-\mathrm{C}_{6} \mathrm{H}_{8} \mathrm{O}_{4}\right]^{+}\end{array}$ & Integriamide & $\mathrm{CG}, \mathrm{MCG}_{12}, \mathrm{MCG}_{20}$ & a \\
\hline 10 & 0.81 & $\mathrm{C}_{25} \mathrm{H}_{30} \mathrm{O}_{12}$ & 538.1677 & 538.1686 & -1.7 & $\begin{array}{l}381.0788\left[\mathrm{M}-\mathrm{CH}_{3}-\mathrm{C}_{7} \mathrm{H}_{10} \mathrm{O}_{3}\right]^{+} ; 366.0930[\mathrm{M}- \\
\left.\mathrm{OH}-\mathrm{C}_{8} \mathrm{H}_{10} \mathrm{O}_{3}\right]^{+} ; 366.0930\left[\mathrm{M}-\mathrm{C}_{19} \mathrm{H}_{21} \mathrm{O}_{8}\right]^{+}\end{array}$ & Linearoside & $\mathrm{CG}, \mathrm{MCG}_{12}, \mathrm{MCG}_{20}$ & [26] \\
\hline 11 & 0.82 & $\mathrm{C}_{9} \mathrm{H}_{11} \mathrm{NO}_{2}$ & 165.0782 & 165.0790 & -0.5 & $\begin{array}{l}\text { 166.0862[M }+\mathrm{H}]^{+} ; 120.0805[\mathrm{M}-\mathrm{COOH}]^{+} \\
103.0543\left[\mathrm{M}-\mathrm{COOH}-\mathrm{NH}_{2}\right]^{+}\end{array}$ & $\begin{array}{l}\text { Phenylpropionic } \\
\text { acid }\end{array}$ & $\mathrm{CG}, \mathrm{MCG}_{12}, \mathrm{MCG}_{20}$ & $\mathrm{~s}$ \\
\hline 12 & 0.91 & $\mathrm{C}_{6} \mathrm{H}_{9} \mathrm{~N}_{3} \mathrm{O}_{2}$ & 155.0762 & 155.0695 & -3.5 & $\begin{array}{l}\text { 156.0762[M + H }]^{+} \\
205.0971[\mathrm{M}+\mathrm{H}]^{+} ; 188.0706\left[\mathrm{M}-\mathrm{NH}_{2}\right]^{+}\end{array}$ & Histidine & $\mathrm{CG}, \mathrm{MCG}_{12}, \mathrm{MCG}_{20}$ & $\mathrm{~s}$ \\
\hline 13 & 1.05 & $\mathrm{C}_{11} \mathrm{H}_{12} \mathrm{~N}_{2} \mathrm{O}_{2}$ & 204.0898 & 204.0899 & -0.5 & $\begin{array}{l}143.0723\left[\mathrm{M}-\mathrm{NH}_{2}-\mathrm{COOH}\right]^{+} ; 118.0649\left[\mathrm{M}-\mathrm{NH}_{2}\right. \\
\left.-\mathrm{COOH}-\mathrm{C}_{2} \mathrm{H}_{3}\right]^{+}\end{array}$ & Tryptophan & $\mathrm{CG}, \mathrm{MCG}_{12}, \mathrm{MCG}_{20}$ & $\mathrm{~s}$ \\
\hline 14 & 1.06 & $\mathrm{C}_{6} \mathrm{H}_{14} \mathrm{~N}_{4} \mathrm{O}_{2}$ & 174.1117 & 174.1117 & 0.0 & $175.1190[\mathrm{M}+\mathrm{H}]^{+}$ & Argentine & $\mathrm{CG}, \mathrm{MCG}_{12}, \mathrm{MCG}_{20}$ & [27] \\
\hline 15 & 3.13 & $\mathrm{C}_{25} \mathrm{H}_{28} \mathrm{O}_{4}$ & 392.2009 & 392.1988 & 5.5 & $393.2082[\mathrm{M}+\mathrm{H}]^{+}$ & Glabrol & $\mathrm{CG}, \mathrm{MCG}_{12}, \mathrm{MCG}_{20}$ & [28] \\
\hline 16 & 3.27 & $\mathrm{C}_{25} \mathrm{H}_{24} \mathrm{O}_{5}$ & 404.1646 & 404.1624 & 5.1 & $405.1719[\mathrm{M}+\mathrm{H}]^{+}$ & Puerarol & $\mathrm{CG}, \mathrm{MCG}_{12}, \mathrm{MCG}_{20}$ & [29] \\
\hline 17 & 4.42 & $\mathrm{C}_{27} \mathrm{H}_{38} \mathrm{O}_{6}$ & 458.2716 & 458.2668 & 10.0 & $481.2609[\mathrm{M}+\mathrm{Na}]^{+} ; 436.2642[\mathrm{M}-\mathrm{COOH}]^{+}$ & Lucideric acid & $\mathrm{CG}, \mathrm{MCG}_{12}, \mathrm{MCG}_{20}$ & a \\
\hline 18 & 4.64 & $\mathrm{C}_{36} \mathrm{H}_{58} \mathrm{O}_{8}$ & 618.4107 & 618.4132 & -4.0 & $619.4180[\mathrm{M}+\mathrm{H}]^{+} ; 421.3446[\mathrm{M}-\mathrm{Glc}-\mathrm{OH}]^{+}$ & $\begin{array}{l}\beta \text {-D-Glcopyranosyl } \\
\text { oleanolate }\end{array}$ & $\mathrm{CG}, \mathrm{MCG}_{12}, \mathrm{MCG}_{20}$ & {$[30]$} \\
\hline 19 & 4.89 & $\mathrm{C}_{48} \mathrm{H}_{82} \mathrm{O}_{19}$ & 962.5484 & 962.5450 & 3.3 & $\begin{array}{l}\text { 985.5312[M }+\mathrm{Na}^{+} ; 765.4795[\mathrm{M}-\mathrm{Glc}-\mathrm{OH}]^{+} \\
541.2637\left[\mathrm{M}-\mathrm{Glc}-\mathrm{OH}-\mathrm{C}_{15} \mathrm{H}_{28} \mathrm{O}\right]^{+} ; 421.3463[\mathrm{M} \\
\left.-\mathrm{Glc}-\mathrm{Glc} / \mathrm{Rha}-2 \times \mathrm{OH}^{+}\right]^{+}\end{array}$ & Majoroside F6 & $\mathrm{CG}, \mathrm{MCG}_{12}, \mathrm{MCG}_{20}$ & [31] \\
\hline 20 & 5.21 & $\mathrm{C}_{31} \mathrm{H}_{46} \mathrm{O}_{8}$ & 546.3248 & 546.3193 & 9.7 & $569.3140[\mathrm{M}+\mathrm{Na}]^{+} ; 133.0859\left[\mathrm{M}-\mathrm{C}_{25} \mathrm{H}_{33} \mathrm{O}_{5}\right]^{+}$ & $\begin{array}{c}\text { Methyl } \\
\text { ganoderate G }\end{array}$ & $\mathrm{CG}, \mathrm{MCG}_{12}, \mathrm{MCG}_{20}$ & a \\
\hline
\end{tabular}


Table 2. Cont

\begin{tabular}{|c|c|c|c|c|c|c|c|c|c|}
\hline No. & $t_{R}(\min )$ & Formula & $\begin{array}{l}\text { Calculated } \\
\text { Mass (Da) }\end{array}$ & $\begin{array}{l}\text { Theoretical } \\
\text { Mass (Da) }\end{array}$ & $\begin{array}{l}\text { Mass Error } \\
(\mathrm{ppm})\end{array}$ & $\mathrm{MS}^{\mathrm{E}}$ Fragmentation & Identification & Sources & Ref. \\
\hline 21 & 5.53 & $\mathrm{C}_{35} \mathrm{H}_{48} \mathrm{O}_{9}$ & 612.3344 & 612.3298 & 7.4 & $\begin{array}{l}\text { 613.3416[M + } \mathrm{H}^{+} ; 582.3264\left[\mathrm{M}-\mathrm{OCH}_{3}\right]^{+} \\
526.2986\left[\mathrm{M}-\mathrm{C}_{4} \mathrm{H}_{7} \mathrm{O}_{2}\right]^{+}\end{array}$ & $\begin{array}{l}\text { Cinobufagin } \\
\text { 3-hemisuberate } \\
\text { methyl ester }\end{array}$ & $\mathrm{CG}, \mathrm{MCG}_{12}, \mathrm{MCG}_{20}$ & $\mathrm{a}$ \\
\hline 22 & 5.56 & $\mathrm{C}_{45} \mathrm{H}_{74} \mathrm{O}_{17}$ & 886.4877 & 886.4926 & -5.4 & $\begin{array}{l}909.4769\left[\mathrm{M}+\mathrm{Na}^{+} ; 745.4383\left[\mathrm{M}-\mathrm{CH}_{2} \mathrm{OH}-\right.\right. \\
\left.\mathrm{C}_{6} \mathrm{H}_{10} \mathrm{O}_{2}\right]^{+} ; 729.4136\left[\mathrm{M}-\mathrm{OH}-\mathrm{C}_{8} \mathrm{H}_{14} \mathrm{O}_{2}\right]^{+} ; \\
601.2768\left[\mathrm{M}-2 \times \mathrm{OH}-\mathrm{C}_{16} \mathrm{H}_{26} \mathrm{O}_{4}\right]^{+} ; 431.1870[\mathrm{M}- \\
\left.\text { Glc }-\mathrm{C}_{19} \mathrm{H}_{27} \mathrm{O}_{2}\right]^{+}\end{array}$ & Shatavarin IV & $\mathrm{CG}, \mathrm{MCG}_{12}, \mathrm{MCG}_{20}$ & [32] \\
\hline 23 & 5.64 & $\mathrm{C}_{25} \mathrm{H}_{32} \mathrm{O}_{13}$ & 540.1800 & 540.1843 & 3.0 & $\begin{array}{l}541.1873[\mathrm{M}+\mathrm{H}]^{+} ; 347.0906\left[\mathrm{M}-\mathrm{CH}_{3} \mathrm{O}-\mathrm{C}_{2} \mathrm{H}_{4}-\right. \\
\left.\mathrm{C}_{8} \mathrm{H}_{9} \mathrm{O}_{2}\right]^{+} ; 195.1008\left[\mathrm{M}-\mathrm{Glc}-\mathrm{C}_{2} \mathrm{H}_{4} \mathrm{O}_{2}-\right. \\
\left.\mathrm{C}_{6} \mathrm{H}_{5} \mathrm{O}_{2}\right]^{+}\end{array}$ & Oleuropein & $\mathrm{CG}, \mathrm{MCG}_{12}, \mathrm{MCG}_{20}$ & [33] \\
\hline 24 & 5.65 & $\mathrm{C}_{14} \mathrm{H}_{16} \mathrm{O}_{4}$ & 248.1025 & 248.1049 & -9.7 & $\begin{array}{l}271.0917[\mathrm{M}+\mathrm{Na}]^{+} ; 195.1008\left[\mathrm{M}-\mathrm{C}_{2} \mathrm{H}_{4}-\mathrm{COH}\right]^{+} \\
\text {189.1348[M }-\mathrm{OH}-\mathrm{COOH}]^{+}\end{array}$ & $\begin{array}{l}\text { Isohistiopterosin } \\
\mathrm{A}\end{array}$ & $\mathrm{CG}, \mathrm{MCG}_{12}, \mathrm{MCG}_{20}$ & a \\
\hline 25 & 5.79 & $\mathrm{C}_{45} \mathrm{H}_{74} \mathrm{O}_{17}$ & 886.4881 & 886.4926 & -5.0 & $\begin{array}{l}\text { 909.4773[M + Na }]^{+} ; 707.4360\left[\mathrm{M}-\mathrm{Glc}^{+} ; 689.4262[\mathrm{M}\right. \\
-\mathrm{Glc}-\mathrm{OH}]^{+} ; 657.3636[\mathrm{M}-\mathrm{Glc}-\mathrm{OH}-2 \times \\
\left.\mathrm{CH}_{3}\right]^{+} ; 609.3646\left[\mathrm{M}-\mathrm{Glc}-\mathrm{C}_{6} \mathrm{H}_{10} \mathrm{O}_{2}\right]^{+} ; 523.3626[\mathrm{M} \\
\left.-\mathrm{Glc}_{\mathrm{Glc}}-\mathrm{C}_{3} \mathrm{H}_{6}\right]^{+}\end{array}$ & $\begin{array}{l}\text { Malonylginsenoside } \\
\text { Rf }\end{array}$ & $\mathrm{CG}, \mathrm{MCG}_{12},{ }^{\# \#} \mathrm{MCG}_{20}$ & [34] \\
\hline 26 & 6.21 & $\mathrm{C}_{42} \mathrm{H}_{70} \mathrm{O}_{14}$ & 798.4778 & 798.4766 & 1.6 & $\begin{array}{l}799.4851[\mathrm{M}+\mathrm{H}]^{+} ; 439.3563[\mathrm{M}-\mathrm{Glc} / \mathrm{Rha}-2 \times \\
\mathrm{OH}]^{+} ; 421.3441\left[\mathrm{M}-\mathrm{Glc} / \mathrm{Rha}-3 \times \mathrm{OH}-\mathrm{H}_{2} \mathrm{O}\right]^{+}\end{array}$ & Ginsenoside $\operatorname{Rg}_{8}$ & $\mathrm{CG}, \mathrm{MCG}_{12}, \mathrm{MCG}_{20}$ & [34] \\
\hline 27 & 6.23 & $\mathrm{C}_{22} \mathrm{H}_{32} \mathrm{O}_{13}$ & 504.1840 & 504.1843 & -0.6 & $\begin{array}{l}\text { 503.1767[M - } \mathrm{H}^{-} ; 457.1715\left[\mathrm{M}-\mathrm{OH}-\mathrm{CH}_{2} \mathrm{OH}\right]^{-} \\
\left.\text {293.0878[M }-\mathrm{C}_{2} \mathrm{H}_{4} \mathrm{O}-\mathrm{CH}_{2} \mathrm{OH}-\mathrm{C}_{8} \mathrm{H}_{9} \mathrm{O}_{2}\right]^{-}\end{array}$ & Cistanoside $\mathrm{H}$ & $\mathrm{CG}, \mathrm{MCG}_{12}, \mathrm{MCG}_{20}$ & [35] \\
\hline 28 & 6.24 & $\mathrm{C}_{23} \mathrm{H}_{28} \mathrm{O}_{11}$ & 480.1594 & 480.1632 & -7.7 & $481.1667[\mathrm{M}+\mathrm{H}]^{+} ; 317.0803\left[\mathrm{M}-\mathrm{C}_{10} \mathrm{H}_{12} \mathrm{O}_{2}\right]^{+}$ & Peoniflorin & $\mathrm{CG}, \mathrm{MCG}_{12}, \mathrm{MCG}_{20}$ & a \\
\hline 29 & 6.28 & $\mathrm{C}_{24} \mathrm{H}_{30} \mathrm{O}_{12}$ & 510.1702 & 510.1737 & -6.9 & $511.1775[\mathrm{M}+\mathrm{H}]^{+} ; 317.0803\left[\mathrm{M}-\mathrm{C}_{11} \mathrm{H}_{14} \mathrm{O}_{3}\right]^{+}$ & Mudanpioside D & $\mathrm{CG}, \mathrm{MCG}_{12}, \mathrm{MCG}_{20}$ & [36] \\
\hline 30 & 6.39 & $\mathrm{C}_{54} \mathrm{H}_{92} \mathrm{O}_{24}$ & 1124.5943 & 1124.5978 & -3.1 & $\begin{array}{l}\text { 1147.5835[M + Na }]^{+} ; 585.2870\left[\mathrm{M}-\mathrm{C}_{25} \mathrm{H}_{47} \mathrm{O}_{12}\right]^{+} \\
\left.\text {325.1130[M }-\mathrm{C}_{42} \mathrm{H}_{71} \mathrm{O}_{14}\right]^{+} \\
\text {985.5302[M }+\mathrm{Na}^{+} ; 865.4789\left[\mathrm{M}-\mathrm{C}_{6} \mathrm{H}_{9} \mathrm{O}\right]^{+}\end{array}$ & Ginsenoside V & $\mathrm{CG}, \mathrm{MCG}_{12}, \mathrm{MCG}_{20}$ & [31] \\
\hline 31 & 6.49 & $\mathrm{C}_{48} \mathrm{H}_{82} \mathrm{O}_{19}$ & 962.5484 & 962.5450 & 3.3 & $\begin{array}{l}\left.\text { 823.4787[M }-\mathrm{C}_{8} \mathrm{H}_{11} \mathrm{O}_{2}\right]^{+} ; 805.4668\left[\mathrm{M}-\mathrm{C}_{8} \mathrm{H}_{13} \mathrm{O}_{3}\right]^{+} \\
555.2763\left[\mathrm{M}-\mathrm{C}_{12} \mathrm{H}_{26} \mathrm{O}_{5}\right]^{+} ; 423.3602[\mathrm{M}-\mathrm{Glc}- \\
\mathrm{Glc} / \mathrm{Glc}-\mathrm{OH}]^{+} ; 405.3507[\mathrm{M}-\mathrm{Glc}-\mathrm{Glc} / \mathrm{Glc}-2 \times \\
\mathrm{OH}]^{+}\end{array}$ & Ginsenoside $\operatorname{Re}_{1}$ & $\begin{array}{l}\mathrm{CG}, \mathrm{MCG}_{12}, \# \#, * \\
\quad \mathrm{MCG}_{20}\end{array}$ & [37] \\
\hline 32 & 6.59 & $\mathrm{C}_{23} \mathrm{H}_{28} \mathrm{O}_{11}$ & 480.1587 & 480.1632 & -9.3 & $481.1660[\mathrm{M}+\mathrm{H}]^{+} ; 317.0810\left[\mathrm{M}-\mathrm{C}_{7} \mathrm{H}_{5} \mathrm{O}-\mathrm{C}_{3} \mathrm{H}_{5} \mathrm{O}\right]^{+}$ & Mudanpioside I & $\mathrm{CG}, \mathrm{MCG}_{12}, \mathrm{MCG}_{20}$ & [38] \\
\hline 33 & 6.64 & $\mathrm{C}_{41} \mathrm{H}_{70} \mathrm{O}_{14}$ & 786.4762 & 786.4766 & -0.4 & $\begin{array}{l}\text { 831.4744[M + } \mathrm{HCOO}^{-} ; 653.4270[\mathrm{M}-\mathrm{H}- \\
\left.\mathrm{C}_{5} \mathrm{H}_{8} \mathrm{O}_{4}\right]^{-}, 491.3710\left[\mathrm{M}-\mathrm{H}-\mathrm{C}_{11} \mathrm{H}_{18} \mathrm{O}_{9}\right]^{-}\end{array}$ & $\begin{array}{l}\text { Notoginsenoside } \\
\text { Rw2 }\end{array}$ & $\mathrm{CG}, \mathrm{MCG}_{12}, \mathrm{MCG}_{20}$ & [39] \\
\hline 34 & 6.67 & $\mathrm{C}_{47} \mathrm{H}_{80} \mathrm{O}_{18}$ & 932.5335 & 932.5345 & -1.0 & $\begin{array}{l}\text { 977.5317[M + } \mathrm{HCOO}^{-} ; 785.4693[\mathrm{M}-\mathrm{Ara}- \\
\left.\mathrm{CH}_{3}\right]^{-} ; 653.4282\left[\mathrm{M}-\mathrm{Glc}-2 \times \mathrm{OH}-\mathrm{C}_{5} \mathrm{H}_{9}\right]^{-}\end{array}$ & Quinquenoside $\mathrm{F}_{6}$ & $\mathrm{CG}, \mathrm{MCG}_{12}, \mathrm{MCG}_{20}$ & [37] \\
\hline 35 & 6.77 & $\mathrm{C}_{36} \mathrm{H}_{60} \mathrm{O}_{9}$ & 636.4217 & 636.4237 & -3.1 & $\begin{array}{l}637.4290[\mathrm{M}+\mathrm{H}]^{+} ; 621.42740[\mathrm{M}-\mathrm{OH}]^{+} ; 423.3605[\mathrm{M} \\
-\mathrm{Glc}-2 \times \mathrm{OH}]^{+}\end{array}$ & Ginsenoside $\mathrm{Rh}_{8}$ & $\mathrm{CG}, \mathrm{MCG}_{12}, \mathrm{MCG}_{20}$ & [40] \\
\hline 36 & 6.84 & $\mathrm{C}_{48} \mathrm{H}_{82} \mathrm{O}_{19}$ & 962.5469 & 962.5450 & 1.9 & $\begin{array}{l}\text { 1007.5456[M + } \mathrm{HCOO}^{-} ; 799.4848\left[\mathrm{M}-\mathrm{Glc}^{-}\right. \\
\text {637.4317[M }-\mathrm{Glc} / \mathrm{Glc}]^{-} ; 179.0545[\mathrm{Glc}-\mathrm{H}]^{-} \\
\text {805.4665[M + Na }]^{+} ; 765.4734[\mathrm{M}-\mathrm{OH}]^{+} ; 677.4220[\mathrm{M}\end{array}$ & $\begin{array}{l}20-\beta \text {-D-Glucopyranos } \\
\text { Rf }\end{array}$ & syl-ginseñ ${ }_{\mathrm{MC}} \mathrm{G}_{12}, \mathrm{MCG}_{20}$ & [41] \\
\hline 37 & 6.80 & $\mathrm{C}_{42} \mathrm{H}_{70} \mathrm{O}_{13}$ & 782.4773 & 782.4816 & -5.4 & $\begin{array}{l}\left.-2 \times \mathrm{OH}-\mathrm{C}_{4} \mathrm{H}_{7} \mathrm{O}\right]^{+} ; 661.4265[\mathrm{M}-3 \times \mathrm{OH}- \\
\left.\mathrm{C}_{4} \mathrm{H}_{7} \mathrm{O}\right]^{+} ; 439.3562[\mathrm{M}-\mathrm{Glc}-\mathrm{Man}-\mathrm{OH}]^{+}\end{array}$ & Ginsenoside $\mathrm{Rh}_{14}$ & $\mathrm{CG}, \mathrm{MCG}_{12}, \mathrm{MCG}_{20}$ & [40] \\
\hline
\end{tabular}


Table 2. Cont

\begin{tabular}{|c|c|c|c|c|c|c|c|c|c|}
\hline No. & $t_{R}(\min )$ & Formula & $\begin{array}{l}\text { Calculated } \\
\text { Mass (Da) }\end{array}$ & $\begin{array}{l}\text { Theoretical } \\
\text { Mass (Da) }\end{array}$ & $\begin{array}{l}\text { Mass Error } \\
(\mathrm{ppm})\end{array}$ & MS $^{\mathrm{E}}$ Fragmentation & Identification & Sources & Ref. \\
\hline 38 & 6.82 & $\mathrm{C}_{17} \mathrm{H}_{24} \mathrm{O}_{8}$ & 356.1460 & 356.1472 & -3.1 & $\begin{array}{l}\text { 379.1352[M + Na }]^{+} ; 145.0495\left[\mathrm{M}-\mathrm{OH}-\mathrm{C}_{11} \mathrm{H}_{13} \mathrm{O}_{3}\right]^{+} \\
\text {977.5392[M + HCOO }]^{-} ; 799.4825[\mathrm{M}-\mathrm{Xyl}]^{-}\end{array}$ & Erigeside II & $\mathrm{CG}, \mathrm{MCG}_{12}, \mathrm{MCG}_{20}$ & [42] \\
\hline 39 & 6.96 & $\mathrm{C}_{47} \mathrm{H}_{80} \mathrm{O}_{18}$ & 932.5410 & 932.5345 & 6.7 & $\begin{array}{l}\text { 769.4724[M }-\mathrm{H}-\mathrm{Glc}^{-} ; 637.4291[\mathrm{M}-(\mathrm{Glc} / \mathrm{Xyl})]^{-} \\
179.0539[\mathrm{Glc}-\mathrm{H}]^{-}\end{array}$ & $\begin{array}{l}\text { Notoginsenoside } \\
\mathrm{R}_{1}\end{array}$ & $\mathrm{CG}, \mathrm{MCG}_{12}, \mathrm{MCG}_{20}$ & $\mathrm{~s}$ \\
\hline 40 & 6.99 & $\mathrm{C}_{28} \mathrm{H}_{44} \mathrm{O}_{12}$ & 572.2810 & 572.2833 & -3.9 & $\begin{array}{l}573.2883[\mathrm{M}+\mathrm{H}]^{+} ; 555.2779[\mathrm{M}-\mathrm{OH}]^{+} ; 531.2860[\mathrm{M} \\
\left.-\mathrm{C}_{2} \mathrm{H}_{3} \mathrm{O}\right]^{+}\end{array}$ & Picrasinoside G & $\mathrm{CG}, \mathrm{MCG}_{12}, \mathrm{MCG}_{20}$ & a \\
\hline 41 & 7.05 & $\mathrm{C}_{48} \mathrm{H}_{82} \mathrm{O}_{19}$ & 962.5425 & 962.5450 & -2.6 & $\begin{array}{l}\text { 1007.5415[M + HCOO }]^{-} ; 799.4822\left[\mathrm{M}-\mathrm{Glc}^{-}\right. \\
\text {637.4333[M - (Glc/Glc) }]^{-}\end{array}$ & $\begin{array}{l}\text { Notoginsenoside } \\
\text { N }\end{array}$ & $\mathrm{CG}, \mathrm{MCG}_{12}, \mathrm{MCG}_{20}$ & [43] \\
\hline 42 & 7.20 & $\mathrm{C}_{48} \mathrm{H}_{82} \mathrm{O}_{19}$ & 962.5422 & 962.5450 & -2.9 & $\begin{array}{l}985.5314[\mathrm{M}+\mathrm{Na}]^{+} ; 703.4371[\mathrm{M}-\mathrm{Glc}-2 \times \mathrm{OH}- \\
\left.\mathrm{CH}_{2} \mathrm{OH}\right]^{-} ; 439.3565[\mathrm{M}-\mathrm{Glc}-\mathrm{Glc} / \mathrm{Glc}-\mathrm{OH}]^{-}\end{array}$ & Ginsenoside $\operatorname{Re}_{2}$ & $\begin{array}{l}\mathrm{CG}, \mathrm{MCG}_{12} \\
\# \#, * * \mathrm{MCG}_{20}\end{array}$ & [40] \\
\hline 43 & 7.34 & $\mathrm{C}_{42} \mathrm{H}_{72} \mathrm{O}_{14}$ & 800.4934 & 800.4922 & 1.4 & $\begin{array}{l}\text { 845.4916[M + } \mathrm{HCOO}^{-} ; 637.4344\left[\mathrm{M}-\mathrm{Glc}^{-}\right. \\
475.3798\left[\mathrm{M}-\mathrm{Glc}^{-} \mathrm{Glc}^{-} ; 179.0553[\mathrm{Glc}-\mathrm{H}]^{-}\right. \\
\text {991.5506[M + HCOO }]^{-} ; 783.4912[\mathrm{M}-\mathrm{Glc}]^{-}\end{array}$ & Ginsenoside $\operatorname{Rg}_{1}$ & $\mathrm{CG}, \mathrm{MCG}_{12}, \mathrm{MCG}_{20}$ & $\mathrm{~s}$ \\
\hline 44 & 7.36 & $\mathrm{C}_{48} \mathrm{H}_{82} \mathrm{O}_{18}$ & 946.5524 & 946.5501 & 2.3 & $\begin{array}{l}\text { 637.4344[M - (Glc/Rha) }]^{-} ; 475.3798[\mathrm{M}-\text { Glc }- \\
(\text { Glc/Rha) }]^{-}\end{array}$ & Ginsenoside Re & $\mathrm{CG}, \mathrm{MCG}_{12}, \mathrm{MCG}_{20}$ & $\mathrm{~s}$ \\
\hline 45 & 7.74 & $\mathrm{C}_{45} \mathrm{H}_{74} \mathrm{O}_{17}$ & 886.4925 & 886.4926 & -0.1 & $\begin{array}{l}885.4853\left[\mathrm{M}-\mathrm{H}^{-} ; 781.4740[\mathrm{M}-\right. \\
\left.\mathrm{HOCOCH}{ }_{2} \mathrm{COOH}\right]^{-} ; 619.4197[\mathrm{M}-\mathrm{Glc}(\mathrm{Mal})]^{-} \\
\left.\text {161.0438[Glc }-\mathrm{H}_{2} \mathrm{O}\right]^{-}\end{array}$ & $\begin{array}{l}\text { Malonylginsenoside } \\
\quad \operatorname{Rg}_{1}\end{array}$ & $\mathrm{CG}, \mathrm{MCG}_{12}, \mathrm{MCG}_{20}$ & [39] \\
\hline 46 & 7.93 & $\mathrm{C}_{48} \mathrm{H}_{76} \mathrm{O}_{19}$ & 956.4960 & 956.4981 & -2.2 & $\begin{array}{l}979.4852\left[\mathrm{M}+\mathrm{Na}^{+} ; 799.4161\left[\mathrm{M}-\mathrm{CO}_{2}-\mathrm{CH}_{2} \mathrm{OH}-\right.\right. \\
\left.\mathrm{C}_{6} \mathrm{H}_{12}\right]^{+} ; 641.4008\left[\mathrm{M}-\mathrm{Glc}-\mathrm{C}_{4} \mathrm{H}_{6} \mathrm{O}_{5}\right]^{+} ; 439.3562[\mathrm{M} \\
-\mathrm{Glc}-\mathrm{Glc} / \mathrm{Glc}(\mathrm{mal})]^{+} ; 145.0493[\mathrm{Glc}-\mathrm{OH}]^{+}\end{array}$ & $\begin{array}{l}\text { Isomer of } \\
\text { ginsenoside Ro }\end{array}$ & ${ }^{\#} \mathrm{CG}, \mathrm{MCG}_{12}, \mathrm{MCG}_{20}$ & [31] \\
\hline 47 & 8.04 & $\mathrm{C}_{51} \mathrm{H}_{84} \mathrm{O}_{21}$ & 1032.5532 & 1032.5505 & 2.6 & $\begin{array}{l}\text { 1031.5460[M - H }]^{-} ; 987.5564\left[\mathrm{M}-\mathrm{CO}_{2}\right]^{-} ; \\
\text {927.5337[M }-\mathrm{HOCOCH} 2 \mathrm{COOH}]^{-} ; 781.4759[\mathrm{M}- \\
\text { Rha(Mal) }]^{-} ; 619.4222\left[\mathrm{M}-(\mathrm{Rha}(\mathrm{Mal}) / \mathrm{Glc}]^{-}\right.\end{array}$ & $\begin{array}{l}\text { Malonylginsenoside } \\
\text { Re }\end{array}$ & $\mathrm{CG}, \mathrm{MCG}_{12}, \mathrm{MCG}_{20}$ & [39] \\
\hline 48 & 8.08 & $\mathrm{C}_{48} \mathrm{H}_{76} \mathrm{O}_{19}$ & 956.4950 & 956.4981 & -3.1 & $\begin{array}{l}\text { 979.4842[M + Na }]^{+} ; 817.4311\left[\mathrm{M}-\mathrm{Glc}^{+} ; 439.3571[\mathrm{M}\right. \\
-\mathrm{Glc} / \mathrm{Glc}-\mathrm{Glc}-\mathrm{OH}]^{+} \\
\text {841.4959[M }-\mathrm{H}]^{-} ; 799.4861\left[\mathrm{M}-\mathrm{CH}_{2} \mathrm{O}\right]^{+} \\
781.4741\left[\mathrm{M}-\mathrm{CH}_{2} \mathrm{O}-\mathrm{OH}\right]^{+} ; 637.4316[\mathrm{M}-\end{array}$ & $\begin{array}{l}\text { Isomer of } \\
\text { ginsenoside Ro }\end{array}$ & $\mathrm{CG}, \mathrm{MCG}_{12}, \mathrm{MCG}_{20}$ & [44] \\
\hline 49 & 8.09 & $\mathrm{C}_{44} \mathrm{H}_{74} \mathrm{O}_{15}$ & 842.5032 & 842.5028 & 0.5 & $\begin{array}{l}\mathrm{Xyl}(\mathrm{mal})]^{+} ; 619.4228[\mathrm{M}-\mathrm{Xyl}(\mathrm{mal})-\mathrm{OH}]^{+} \\
475.3798\left[\mathrm{M}-\mathrm{Xyl}(\mathrm{mal})-\mathrm{Glc}^{+} ; 179.0550[\mathrm{Glc}-\mathrm{H}]^{+}\right. \\
161.0439[\mathrm{Glc}-\mathrm{OH}]^{+}\end{array}$ & Yesanchinoside D & $\mathrm{CG}, \mathrm{MCG}_{12}, \mathrm{MCG}_{20}$ & [45] \\
\hline 50 & 8.10 & $\mathrm{C}_{45} \mathrm{H}_{74} \mathrm{O}_{17}$ & 886.4931 & 886.4926 & 0.6 & $\begin{array}{l}\text { 885.4858[M }-\mathrm{H}]^{-} ; 781.4741[\mathrm{M}-\mathrm{H}- \\
\mathrm{HOCOCH} \mathrm{COOH}^{-} ; 619.4228[\mathrm{M}-\mathrm{H}-\mathrm{Glc}(\mathrm{Mal})]^{-} \\
\left.\text {161.0439[Glc }-\mathrm{H}-\mathrm{H}_{2} \mathrm{O}\right]^{-}\end{array}$ & $\begin{array}{l}\text { Isomer of } \\
\text { malonylginsenoside } \\
\operatorname{Rg}_{1}\end{array}$ & $\mathrm{CG}, \mathrm{MCG}_{12}, \mathrm{MCG}_{20}$ & [39] \\
\hline 51 & 8.49 & $\mathrm{C}_{41} \mathrm{H}_{70} \mathrm{O}_{13}$ & 770.4801 & 770.4816 & -1.5 & $815.4784\left[\mathrm{M}+\mathrm{HCOO}^{-} ; 637.4321[\mathrm{M}-\mathrm{Xyl}]^{-}\right.$ & $\begin{array}{l}\text { Notoginsenoside } \\
\mathrm{R}_{2}\end{array}$ & $\mathrm{CG}, \mathrm{MCG}_{12}, \mathrm{MCG}_{20}$ & [39] \\
\hline 52 & 8.50 & $\mathrm{C}_{56} \mathrm{H}_{94} \mathrm{O}_{24}$ & 1150.6124 & 1150.6135 & -1.1 & $\begin{array}{l}1149.6051[\mathrm{M}-\mathrm{H}]^{-} ; 1119.5951\left[\mathrm{M}-\mathrm{CH}_{2} \mathrm{OH}-2 \times\right. \\
\mathrm{OH}]^{-} ; 807.4861[\mathrm{M}-\mathrm{Glc} / \mathrm{Glc}-\mathrm{OH}]^{-} ; 605.4423[\mathrm{M} \\
-\mathrm{Glc} / \mathrm{Glc}-\mathrm{Glc}(\mathrm{mal})]^{-} ; 325.1119[\mathrm{Glc} / \mathrm{Glc}-\mathrm{OH}]^{-}\end{array}$ & $\begin{array}{l}\text { Quinquenoside } \\
\mathrm{R}_{1}\end{array}$ & $\mathrm{CG}, \mathrm{MCG}_{12},{ }^{\# \#} \mathrm{MCG}_{20}$ & [46] \\
\hline
\end{tabular}


Table 2. Cont

\begin{tabular}{|c|c|c|c|c|c|c|c|c|c|}
\hline No. & $t_{R}(\min )$ & Formula & $\begin{array}{l}\text { Calculated } \\
\text { Mass (Da) }\end{array}$ & $\begin{array}{l}\text { Theoretical } \\
\text { Mass (Da) }\end{array}$ & $\begin{array}{l}\text { Mass Error } \\
\quad(\mathrm{ppm})\end{array}$ & $\mathrm{MS}^{\mathrm{E}}$ Fragmentation & Identification & Sources & Ref. \\
\hline 53 & 8.60 & $\mathrm{C}_{22} \mathrm{H}_{30} \mathrm{O}_{47}$ & 406.1957 & 406.1992 & -8.5 & $407.2030[\mathrm{M}+\mathrm{H}]^{+} ; 376.1859\left[\mathrm{M}-\mathrm{OCH}_{3}\right]^{+}$ & Nigakilactone $\mathrm{K}$ & $\mathrm{CG}, \mathrm{MCG}_{12}, \mathrm{MCG}_{20}$ & [47] \\
\hline 54 & 8.87 & $\mathrm{C}_{48} \mathrm{H}_{82} \mathrm{O}_{19}$ & 962.5445 & 962.5450 & -0.5 & $1007.5427\left[\mathrm{M}+\mathrm{HCOO}^{-} ; 797.4706\left[\mathrm{M}-\mathrm{Glc}^{-}\right.\right.$ & Ginsenoside $\mathrm{Re}_{3}$ & $\mathrm{CG}, \mathrm{MCG}_{12}, \mathrm{MCG}_{20}$ & [37] \\
\hline 55 & 8.96 & $\mathrm{C}_{56} \mathrm{H}_{92} \mathrm{O}_{25}$ & 1164.5929 & 1164.5928 & 0.1 & $\begin{array}{l}1187.5821[\mathrm{M}+\mathrm{Na}]^{+} ; 1147.5803[\mathrm{M}-\mathrm{OH}]^{+} \\
805.4305\left[\mathrm{M}-\mathrm{Ara} / \mathrm{Glc}-\mathrm{CH}_{2} \mathrm{OH}-\mathrm{CH}_{3}\right]^{+} \\
443.3868[\mathrm{M}-\mathrm{Ara} / \mathrm{Glc}-\mathrm{Glc} / \mathrm{Glc}(\mathrm{mal})]^{+}\end{array}$ & $\begin{array}{l}\text { Malonylginsenoside } \\
\mathrm{Rb}_{2}\end{array}$ & $\begin{array}{c}\mathrm{CG}, \mathrm{MCG}_{12}, \# \#, * \\
\mathrm{MCG}_{20}\end{array}$ & [44] \\
\hline 56 & 9.41 & $\mathrm{C}_{59} \mathrm{H}_{100} \mathrm{O}_{27}$ & 1240.6488 & 1240.6452 & 2.8 & $\begin{array}{l}\text { 1285.6740[M + } \mathrm{HCOO}^{-} ; 945.5421[\mathrm{M}-(\mathrm{Ara} / \mathrm{Xyl}) \\
]^{-} ; 913.5184[\mathrm{M}-(\mathrm{Glc} / \mathrm{Glc})]^{-} ; 783.4900[\mathrm{M}- \\
(\mathrm{Ara} / \mathrm{Xyl})-\mathrm{Glc}]^{-}\end{array}$ & $\begin{array}{l}\text { Notoginsenoside } \\
\mathrm{R}_{4}\end{array}$ & $\mathrm{CG}, \mathrm{MCG}_{12}, \mathrm{MCG}_{20}$ & $\mathrm{~s}$ \\
\hline 57 & 9.56 & $\mathrm{C}_{42} \mathrm{H}_{72} \mathrm{O}_{14}$ & 800.4921 & 800.4922 & -0.1 & $\begin{array}{l}\text { 845.4903[M + } \mathrm{HCOO}^{-} ; 637.4319\left[\mathrm{M}-\mathrm{Glc}^{-}\right. \\
\text {475.3786[M }-(\mathrm{Glc} / \mathrm{Glc})]^{-} ; 1,3 \mathrm{~A}_{2 \beta} 221.0658 \\
\left.\text { 161.0439[Glc }-\mathrm{H}-\mathrm{H}_{2} \mathrm{O}\right]^{-} ; 2,5 \mathrm{~A}_{1 \beta} 101.0235\end{array}$ & Ginsenoside Rf & $\mathrm{CG}, \mathrm{MCG}_{12}, \mathrm{MCG}_{20}$ & $\mathrm{~s}$ \\
\hline 58 & 9.79 & $\mathrm{C}_{18} \mathrm{H}_{34} \mathrm{O}_{5}$ & 330.2398 & 330.2406 & -2.3 & $\begin{array}{l}353.2290[\mathrm{M}+\mathrm{Na}]^{+} ; 213.1459[\mathrm{M}+\mathrm{H}-\mathrm{COOH}- \\
\left.\mathrm{C}_{5} \mathrm{H}_{11}\right]^{+}\end{array}$ & $\begin{array}{l}12,13,15 \text {-Trihydroxy- } 9 \\
\text { acid }\end{array}$ & -pctadecenoic $, \mathrm{MCG}_{12}, \mathrm{MCG}_{20}$ & [48] \\
\hline 59 & 9.87 & $\mathrm{C}_{41} \mathrm{H}_{70} \mathrm{O}_{13}$ & 770.4809 & 770.4816 & -1.0 & $\begin{array}{l}\text { 815.4791[M }+\mathrm{HCOO}^{-} ; 475.3783[\mathrm{M}-(\mathrm{Glc} / \mathrm{Xyl})]^{-} \\
\left.\text {161.0437[Glc }-\mathrm{H}-\mathrm{H}_{2} \mathrm{O}\right]^{-}\end{array}$ & Ginsenoside $F_{5}$ & $\mathrm{CG}, \mathrm{MCG}_{12}, \mathrm{MCG}_{20}$ & $\mathrm{~s}$ \\
\hline 60 & 9.89 & $\mathrm{C}_{60} \mathrm{H}_{102} \mathrm{O}_{28}$ & 1270.6635 & 1270.6558 & 5.9 & $\begin{array}{l}\text { 1315.6617[M }+\mathrm{HCOO}^{-} ; 841.4991[\mathrm{M}-\mathrm{Glc} / \mathrm{Glc}- \\
\left.\mathrm{OH}-\mathrm{C}_{4} \mathrm{H}_{4}\right]^{-} ; 769.4777\left[\mathrm{M}-\mathrm{Glc} / \mathrm{Glc} / \mathrm{Glc}-\mathrm{CH}_{3}\right]^{-} \\
1255.6340[\mathrm{M}+\mathrm{HCOO}]^{-} ; 1077.5833[\mathrm{M}-\mathrm{Xyl}]^{-}\end{array}$ & Ginsenoside $\mathrm{Ra}_{0}$ & $\mathrm{CG}, \mathrm{MCG}_{12}, \mathrm{MCG}_{20}$ & [49] \\
\hline 61 & 9.94 & $\mathrm{C}_{58} \mathrm{H}_{98} \mathrm{O}_{26}$ & 1210.6358 & 1210.6346 & 1.0 & $\begin{array}{l}1047.5719[\mathrm{M}-\mathrm{Glc}]^{-} ; 955.4871[\mathrm{M}-\mathrm{Glc}-\mathrm{OH}- \\
\left.\mathrm{C}_{4} \mathrm{H}_{7}\right]^{-} ; 783.4892[\mathrm{M}-\mathrm{Glc} / \mathrm{Xyl} / \mathrm{Rha}]^{-} \\
469.1084[\mathrm{M}+\mathrm{Na}]^{+} ; 429.1154[\mathrm{M}-\mathrm{OH}]^{+} ; 385.0884[\mathrm{M}\end{array}$ & Ginsenoside $\mathrm{Ra}_{2}$ & $\mathrm{CG}, \mathrm{MCG}_{12}, \mathrm{MCG}_{20}$ & [50] \\
\hline 62 & 10.00 & $\mathrm{C}_{22} \mathrm{H}_{22} \mathrm{O}_{10}$ & 446.1192 & 446.1213 & -4.5 & $\begin{array}{l}\left.-\mathrm{OH}-\mathrm{CH}_{3}-\mathrm{CH}_{2} \mathrm{OH}\right]^{+} ; 341.0661\left[\mathrm{M}-\mathrm{C}_{4} \mathrm{H}_{8} \mathrm{O}_{3}\right]^{+} \\
237.0746\left[\mathrm{M}-\mathrm{C}_{10} \mathrm{H}_{13} \mathrm{O}_{5}\right]^{+} ; 193.0483[\mathrm{M}- \\
\left.\mathrm{C}_{12} \mathrm{H}_{16} \mathrm{O}_{6}\right]^{+}\end{array}$ & Glycitin & $\mathrm{CG}, \mathrm{MCG}_{12}, \mathrm{MCG}_{20}$ & $\mathrm{a}$ \\
\hline 63 & 10.01 & $\mathrm{C}_{59} \mathrm{H}_{100} \mathrm{O}_{27}$ & 1240.6462 & 1240.6452 & 0.8 & 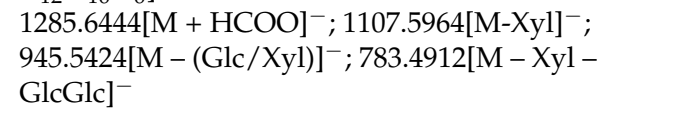 & $\begin{array}{l}\text { Notoginsenoside } \\
\mathrm{Fa}\end{array}$ & $\mathrm{CG}, \mathrm{MCG}_{12}, \mathrm{MCG}_{20}$ & [50] \\
\hline 64 & 10.05 & $\mathrm{C}_{54} \mathrm{H}_{92} \mathrm{O}_{23}$ & 1108.6101 & 1108.6029 & 6.2 & $\begin{array}{l}\text { 1153.6083[M + } \mathrm{HCOO}^{-} ; 945.5437\left[\mathrm{M}-\mathrm{Glc}^{-} ;\right. \\
783.4888[\mathrm{M}-(\mathrm{Glc} / \mathrm{Glc})]^{-} ; 621.4382[\mathrm{M}-(\mathrm{Glc} / \mathrm{Glc})- \\
\mathrm{Glc}^{-} ; 459.3835[\mathrm{M}-(\mathrm{Glc} / \mathrm{Glc})-(\mathrm{Glc} / \mathrm{Glc})]^{-} ; \\
{ }^{2,5} \mathrm{~A}_{1 \beta} 101.0235\end{array}$ & Ginsenoside $\mathrm{Rb}_{1}$ & $\mathrm{CG}, \mathrm{MCG}_{12}, \mathrm{MCG}_{20}$ & $\mathrm{~s}$ \\
\hline 65 & 10.10 & $\mathrm{C}_{42} \mathrm{H}_{70} \mathrm{O}_{12}$ & 766.4863 & 766.4867 & -0.5 & $\begin{array}{l}767.4936[\mathrm{M}+\mathrm{H}]^{+} ; 443.3866[\mathrm{M}-\mathrm{Rha}-\mathrm{Glc}]^{+} \\
425.3762[\mathrm{M}-\mathrm{Rha}-\mathrm{Glc}-\mathrm{OH}]^{+}\end{array}$ & Ginsenoside $\operatorname{Rg}_{4}$ & $\mathrm{CG}, \mathrm{MCG}_{12}, \mathrm{MCG}_{20}$ & [34] \\
\hline 66 & 10.20 & $\mathrm{C}_{57} \mathrm{H}_{94} \mathrm{O}_{26}$ & 1194.6087 & 1194.6033 & 4.5 & $\begin{array}{l}\text { 1193.6015[M - H }]^{-} ; 1149.6098\left[\mathrm{M}-\mathrm{CO}_{2}\right]^{-} \\
783.4908[\mathrm{M}-\mathrm{Glc} / \mathrm{Glc})]^{-} ; 179.0545[\mathrm{Glc}-\mathrm{H}]^{-}\end{array}$ & $\begin{array}{l}\text { Isomer of } \\
\text { malonylginsenoside } \\
\mathrm{Rb}_{1}\end{array}$ & $\begin{array}{l}\mathrm{CG}, \mathrm{MCG}_{12}, \#, \ldots+* * \\
\mathrm{MCG}_{20}\end{array}$ & [39] \\
\hline 67 & 10.22 & $\mathrm{C}_{42} \mathrm{H}_{72} \mathrm{O}_{13}$ & 784.4997 & 784.4973 & 2.9 & $\begin{array}{l}\text { 829.4979[M + } \mathrm{HCOO}^{-} ; 637.4336\left[\mathrm{M}-\mathrm{Rha}^{-}\right. \\
\text {475.3809[M - } \\
\text { (Glc/Rha })]^{-} ; 161.0449\left[^{\mathrm{Rha}}-\mathrm{H}\right]^{-}\end{array}$ & $\begin{array}{l}\text { 20(R)-Ginsenoside } \\
\mathrm{Rg}_{2}\end{array}$ & $\mathrm{CG}, \mathrm{MCG}_{12}, \mathrm{MCG}_{20}$ & $\mathrm{~s}$ \\
\hline 68 & 10.25 & $\mathrm{C}_{36} \mathrm{H}_{62} \mathrm{O}_{9}$ & 638.4407 & 638.4394 & 2.9 & $\begin{array}{l}\text { 683.4389[M + } \mathrm{HCOO}^{-} ; 161.0449[\mathrm{Glc}-\mathrm{H}- \\
\left.\mathrm{H}_{2} \mathrm{O}\right]^{-}\end{array}$ & Ginsenoside $\mathrm{Rh}_{1}$ & $\mathrm{CG}, \mathrm{MCG}_{12}, \mathrm{MCG}_{20}$ & $\mathrm{~s}$ \\
\hline
\end{tabular}


Table 2. Cont.

\begin{tabular}{|c|c|c|c|c|c|c|c|c|c|}
\hline No. & $t_{R}(\min )$ & Formula & $\begin{array}{l}\text { Calculated } \\
\text { Mass (Da) }\end{array}$ & $\begin{array}{l}\text { Theoretical } \\
\text { Mass (Da) }\end{array}$ & $\begin{array}{l}\text { Mass Error } \\
(\mathrm{ppm})\end{array}$ & $\mathrm{MS}^{\mathrm{E}}$ Fragmentation & Identification & Sources & Ref \\
\hline 69 & 10.27 & $\mathrm{C}_{41} \mathrm{H}_{70} \mathrm{O}_{13}$ & 770.4779 & 770.4816 & -4.7 & $\begin{array}{l}\text { 793.4672[M + Na }]^{+} ; 587.4276[\mathrm{M}-\mathrm{Ara}(\mathrm{p})-2 \times \\
\mathrm{OH}]^{+} ; 423.3589[\mathrm{M}-\mathrm{Ara}(\mathrm{p}) / \mathrm{Glc}-2 \times \mathrm{OH}]^{+}\end{array}$ & Ginsenoside $F_{3}$ & $\mathrm{CG}^{,}{ }^{\Delta \Delta} \mathrm{MCG}_{12}, \mathrm{MCG}_{20}$ & [34] \\
\hline 70 & 10.29 & $\mathrm{C}_{36} \mathrm{H}_{60} \mathrm{O}_{8}$ & 620.4292 & 620.4288 & 0.7 & $\begin{array}{l}\text { 621.4365[M + H }]^{+} ; 390.2277\left[\mathrm{M}-\mathrm{C}_{17} \mathrm{H}_{26}\right]^{+} \\
\left.\text {187.1473[M - OH }-\mathrm{Glc}-\mathrm{C}_{16} \mathrm{H}_{24} \mathrm{O}\right]^{+} \\
1101.5805[\mathrm{M}+\mathrm{Na}]^{+} ; 939.5312\left[\mathrm{M}-\mathrm{Glc}^{+}\right.\end{array}$ & Ginsenoside $\mathrm{Rh}_{4}$ & $\mathrm{CG}, \mathrm{MCG}_{12}, \mathrm{MCG}_{20}$ & [40] \\
\hline 71 & 10.32 & $\mathrm{C}_{53} \mathrm{H}_{90} \mathrm{O}_{22}$ & 1078.5939 & 1078.5924 & 1.3 & $\begin{array}{l}\text { 929.5452[M - Ara(f) }]^{+} ; 789.4784[\mathrm{M}-\operatorname{Ara}(\mathrm{f})- \\
\mathrm{Glc}^{+}\end{array}$ & Ginsenoside Rc & $\mathrm{CG}, \mathrm{MCG}_{12}, \mathrm{MCG}_{20}$ & $\mathrm{~s}$ \\
\hline 72 & 10.34 & $\mathrm{C}_{58} \mathrm{H}_{98} \mathrm{O}_{26}$ & 1210.6356 & 1210.6346 & 0.7 & $\begin{array}{l}\text { 1255.6338[M + } \mathrm{HCOO}^{-} ; 1077.5851\left[\mathrm{M}-\mathrm{Xly}^{-}\right. \\
\text {1047.5702[M }-\mathrm{Glc}^{-} ; 945.5396[\mathrm{M}-(\mathrm{Xly} / \\
\mathrm{Ara}(\mathrm{p}))]^{-} ; 621.4323\left[\mathrm{M}-\left(\mathrm{Xly} / \mathrm{Ara}(\mathrm{p}) / \mathrm{Glc}-\mathrm{Glc}^{-}\right.\right.\end{array}$ & Ginsenoside $\mathrm{Ra}_{1}$ & $\mathrm{CG}, \mathrm{MCG}_{12}, \mathrm{MCG}_{20}$ & [50] \\
\hline 73 & 10.38 & $\mathrm{C}_{42} \mathrm{H}_{70} \mathrm{O}_{12}$ & 766.4872 & 766.4867 & 0.6 & $\begin{array}{l}767.4945[\mathrm{M}+\mathrm{H}]^{+} ; 605.4423\left[\mathrm{M}-\mathrm{Glc}^{+} ; 443.3870[\mathrm{M}\right. \\
-\mathrm{Glc} / \mathrm{Xyl}]^{+} ; 407.3660[\mathrm{M}-\mathrm{Glc}-2 \times \mathrm{OH}]^{+} ; \\
163.0591[\mathrm{Glc}-\mathrm{OH}]^{+} ; 145.04901\left[\mathrm{Glc}-\mathrm{OH}-\mathrm{H}_{2} \mathrm{O}\right]^{+}\end{array}$ & Ginsenoside $\operatorname{Rg}_{5}$ & $\mathrm{CG}, \mathrm{MCG}_{12}, \mathrm{MCG}_{20}$ & $\mathrm{~s}$ \\
\hline 74 & 10.47 & $\mathrm{C}_{56} \mathrm{H}_{92} \mathrm{O}_{25}$ & 1164.5947 & 1164.5928 & 1.6 & $\begin{array}{l}1163.5874[\mathrm{M}-\mathrm{H}]^{-} ; 1119.5961\left[\mathrm{M}-\mathrm{CO}_{2}\right]^{-} \\
927.5320\left[\mathrm{M}-\mathrm{Ara}(\mathrm{f})-\mathrm{HOCOCH}_{2} \mathrm{COOH}\right]^{-}\end{array}$ & $\begin{array}{l}\text { Malonylginsenoside } \\
\text { Rc }\end{array}$ & $\mathrm{CG}, \mathrm{MCG}_{12}, \mathrm{MCG}_{20}$ & [44] \\
\hline 75 & 10.51 & $\mathrm{C}_{48} \mathrm{H}_{76} \mathrm{O}_{19}$ & 956.5001 & 956.4981 & 2.1 & $\begin{array}{l}\text { 955.4928[M - H }]^{-} ; 793.4399\left[\mathrm{M}-\mathrm{Glc}^{-}\right. \\
613.3739[\mathrm{M}-\mathrm{Glc}-\mathrm{Glc}-\mathrm{OH}]^{-}\end{array}$ & Ginsenoside Ro & ${ }^{\#} \mathrm{CG}, \mathrm{MCG}_{12}, \mathrm{MCG}_{20}$ & $\mathrm{~s}$ \\
\hline 76 & 10.57 & $\mathrm{C}_{57} \mathrm{H}_{94} \mathrm{O}_{26}$ & 1194.6059 & 1194.6033 & 2.2 & $\begin{array}{l}\text { 1193.5986[M - H }]^{-} ; 1149.6062\left[\mathrm{M}-\mathrm{CO}_{2}\right]^{-} \\
\left.\text {1089.5851[M }-\mathrm{HOCOCH}{ }_{2} \mathrm{COOH}\right]^{-} ; 945.5428[\mathrm{M}- \\
\text { Glc(Mal) }]^{-} ; 783.4926[\mathrm{M}-(\mathrm{Glc} / \mathrm{Glc})]^{-}\end{array}$ & $\begin{array}{l}\text { Malonylginsenoside } \\
\mathrm{Rb}_{1}\end{array}$ & $\mathrm{CG}, \mathrm{MCG}_{12}, \mathrm{MCG}_{20}$ & [39] \\
\hline 77 & 10.63 & $\mathrm{C}_{53} \mathrm{H}_{90} \mathrm{O}_{22}$ & 1078.5979 & 1078.5924 & 4.9 & $\begin{array}{l}\text { 1123.5961[M + } \mathrm{HCOO}^{-} ; 945.5448[\mathrm{M}-\mathrm{Ara}(\mathrm{p})]^{-} \\
783.4896[\mathrm{M}-(\mathrm{Ara} / \mathrm{Glc})]^{-} ; 149.0443[\operatorname{Ara}(\mathrm{p})-\mathrm{H}]^{-}\end{array}$ & $\begin{array}{l}\text { Ginsenoside } \\
\mathrm{Rb}_{2} / \mathrm{Rb}_{3}\end{array}$ & $\mathrm{CG}, \mathrm{MCG}_{12}, \mathrm{MCG}_{20}$ & $\mathrm{~s}$ \\
\hline 78 & 10.77 & $\mathrm{C}_{56} \mathrm{H}_{92} \mathrm{O}_{25}$ & 1164.5986 & 1164.5928 & 5.0 & $\begin{array}{l}\text { 1163.5913[M - H }]^{-} ; 1101.5822\left[\mathrm{M}-\mathrm{CO}_{2}\right]^{-} \\
765.4782[\mathrm{M}-\mathrm{H}-\mathrm{Glc}(\mathrm{Mal})-\operatorname{Ara}(\mathrm{p})-\mathrm{OH}]^{-}\end{array}$ & $\begin{array}{l}\text { Malonylginsenoside } \\
\mathrm{Rb}_{2}\end{array}$ & $\mathrm{CG}, \mathrm{MCG}_{12}, \mathrm{MCG}_{20}$ & [44] \\
\hline 79 & 11.06 & $\mathrm{C}_{36} \mathrm{H}_{62} \mathrm{O}_{9}$ & 638.4391 & 638.4394 & -0.4 & 683.4373[M + $\mathrm{HCOO}]^{-}$ & $\begin{array}{l}\text { 20(R)-Ginsenoside } \\
\mathrm{Rh}_{1}\end{array}$ & $\mathrm{CG}, \mathrm{MCG}_{12}, \mathrm{MCG}_{20}$ & $\mathrm{~s}$ \\
\hline 80 & 11.14 & $\mathrm{C}_{36} \mathrm{H}_{62} \mathrm{O}_{9}$ & 638.4399 & 638.4394 & 0.7 & $661.4291[\mathrm{M}+\mathrm{Na}]^{+} ; 376.2462\left[\mathrm{M}-\mathrm{C}_{17} \mathrm{H}_{24} \mathrm{O}_{2}\right]^{+}$ & Ginsenoside $\mathrm{F}_{1}$ & $\mathrm{CG}, \mathrm{MCG}_{12}, \mathrm{MCG}_{20}$ & $\mathrm{~s}$ \\
\hline 81 & 11.15 & $\mathrm{C}_{56} \mathrm{H}_{92} \mathrm{O}_{25}$ & 1164.5971 & 1164.5928 & 3.7 & $\begin{array}{l}1163.5898[\mathrm{M}-\mathrm{H}]^{-} ; 1119.6000\left[\mathrm{M}-\mathrm{CO}_{2}\right]^{-} \\
\left.\text {1059.5772[M }-\mathrm{H}-\mathrm{C}_{3} \mathrm{H}_{4} \mathrm{O}_{4}\right]^{-}\end{array}$ & $\begin{array}{l}\text { Malonylginsenoside } \\
\mathrm{Rb}_{3}\end{array}$ & $\mathrm{CG}, \mathrm{MCG}_{12}, \mathrm{MCG}_{20}$ & [39] \\
\hline 82 & 11.27 & $\mathrm{C}_{48} \mathrm{H}_{82} \mathrm{O}_{18}$ & 946.5482 & 946.5501 & -1.9 & $\begin{array}{l}\text { 991.5464[M + } \mathrm{HCOO}^{-} ; 783.4878\left[\mathrm{M}-\mathrm{Glc}^{-}\right. \\
621.4350\left[\mathrm{M}-\text { (Glc/Glc }^{-} ; 161.0435[\mathrm{Glc}-\mathrm{H}]^{-}\right.\end{array}$ & Ginsenoside Rd & $\mathrm{CG}, \mathrm{MCG}_{12}, \mathrm{MCG}_{20}$ & $\mathrm{~s}$ \\
\hline 83 & 11.31 & $\mathrm{C}_{55} \mathrm{H}_{92} \mathrm{O}_{23}$ & 1120.6049 & 1120.6029 & 1.7 & $1143.5941[\mathrm{M}+\mathrm{Na}]^{+} ; 831.4874[\mathrm{M}-\mathrm{Glc}(\mathrm{mal})]^{-}$ & Ginsenoside $\mathrm{Rs}_{1}$ & $\begin{array}{l}\mathrm{CG}, \mathrm{MCG}_{12}, \# \#, * \\
\mathrm{MCG}_{20}\end{array}$ & $\mathrm{~s}$ \\
\hline 84 & 11.36 & $\mathrm{C}_{42} \mathrm{H}_{70} \mathrm{O}_{12}$ & 766.4875 & 766.4867 & 1.0 & $\begin{array}{l}\text { 767.4947[M + H }]^{+} ; 605.4423[\mathrm{M}-\mathrm{Rha}]^{+} ; 587.4300[\mathrm{M} \\
-\mathrm{Rha}-\mathrm{OH}]^{+} ; 569.4211[\mathrm{M}-\mathrm{Rha}-2 \times \mathrm{OH}]^{+} \\
443.3866[\mathrm{M}-\mathrm{Rha} / \mathrm{Glc}]^{+} ; 425.3769[\mathrm{M}-\mathrm{Rha} / \mathrm{Glc} \\
-\mathrm{OH}]^{+} ; 145.0491\left[\mathrm{Rha}-\mathrm{H}-\mathrm{H}_{2} \mathrm{O}\right]^{+}\end{array}$ & Ginsenoside $\operatorname{Rg}_{6}$ & $\mathrm{CG}, \mathrm{MCG}_{12}, \mathrm{MCG}_{20}$ & [44] \\
\hline 85 & 11.42 & $\mathrm{C}_{51} \mathrm{H}_{84} \mathrm{O}_{21}$ & 1032.5515 & 1032.5505 & 0.9 & $\begin{array}{l}1131.5442[\mathrm{M}-\mathrm{H}]^{-} ; 765.4785[\mathrm{M}-\mathrm{Glc}(\mathrm{mal})- \\
\mathrm{OH}]^{-} ; 621.4372\left[\mathrm{M}-(\mathrm{Glc} / \mathrm{Glc}(\mathrm{mal})]^{-}\right.\end{array}$ & $\begin{array}{l}\text { Malonylginsenoside } \\
\text { Rd }\end{array}$ & $\mathrm{CG}, \mathrm{MCG}_{12}, \mathrm{MCG}_{20}$ & [45] \\
\hline 86 & 11.53 & $\mathrm{C}_{55} \mathrm{H}_{92} \mathrm{O}_{23}$ & 1120.6065 & 1120.6029 & 3.0 & $\begin{array}{l}1165.6047\left[\mathrm{M}+\mathrm{HCOO}^{-} ; 1077.5851\left[\mathrm{M}-\mathrm{Ac}^{-}\right.\right. \\
1059.5745[\mathrm{M}-\mathrm{Ac}-\mathrm{OH}]^{-}\end{array}$ & Ginsenoside $\mathrm{Rs}_{2}$ & $\mathrm{CG}, \mathrm{MCG}_{12}, \mathrm{MCG}_{20}$ & $\mathrm{~s}$ \\
\hline
\end{tabular}


Table 2. Cont.

\begin{tabular}{|c|c|c|c|c|c|c|c|c|c|}
\hline No. & $t_{R}(\min )$ & Formula & $\begin{array}{l}\text { Calculated } \\
\text { Mass (Da) }\end{array}$ & $\begin{array}{l}\text { Theoretical } \\
\text { Mass (Da) }\end{array}$ & $\begin{array}{l}\text { Mass Error } \\
\quad(\mathrm{ppm})\end{array}$ & $\mathrm{MS}^{\mathrm{E}}$ Fragmentation & Identification & Sources & Ref \\
\hline 87 & 11.69 & $\mathrm{C}_{42} \mathrm{H}_{70} \mathrm{O}_{13}$ & 782.4738 & 782.4816 & -9.7 & $\begin{array}{l}\text { 805.4631[M + Na }]^{+} ; 621.4354[\mathrm{M}-\mathrm{Glc}]^{+} \\
\left.\text {311.0902[Glc/Glc }-\mathrm{CH}_{2} \mathrm{OH}\right]^{+}\end{array}$ & Ginsenoside $\operatorname{Rg}_{10}$ & $\mathrm{CG}, \mathrm{MCG}_{12}, \mathrm{MCG}_{20}$ & $\mathrm{a}$ \\
\hline 88 & 11.79 & $\mathrm{C}_{48} \mathrm{H}_{82} \mathrm{O}_{18}$ & 946.5494 & 946.5501 & -0.7 & $\begin{array}{l}\text { 991.5476[M + } \mathrm{HCOO}^{-} ; 927.5308[\mathrm{M}-\mathrm{OH}]^{-} \\
783.4926\left[\mathrm{M}-\mathrm{Glc}^{-} ; 621.4412\left[\mathrm{M}-\text { (Glc/Glc) }^{-}\right.\right.\end{array}$ & Gypenoside XVII & $\mathrm{CG}, \mathrm{MCG}_{12}, \mathrm{MCG}_{20}$ & $\mathrm{~s}$ \\
\hline 89 & 11.81 & $\mathrm{C}_{51} \mathrm{H}_{84} \mathrm{O}_{21}$ & 1032.5504 & 1032.5505 & -0.1 & $\begin{array}{l}\text { 1031.5431[M }-\mathrm{H}]^{-} ; 987.5535\left[\mathrm{M}-\mathrm{CO}_{2}\right]^{-} \\
621.4412[\mathrm{M}-(\mathrm{Glc} / \mathrm{Glc}(\mathrm{mal}))]^{-} ; 179.0546[\mathrm{Glc}- \\
\mathrm{H}]^{-}\end{array}$ & $\begin{array}{l}\text { Isomer of } \\
\text { malonylginsenoside } \\
\text { Rd }\end{array}$ & $\mathrm{CG}, \mathrm{MCG}_{12}, \mathrm{MCG}_{20}$ & [49] \\
\hline 90 & 11.88 & $\mathrm{C}_{48} \mathrm{H}_{82} \mathrm{O}_{18}$ & 946.5476 & 946.55021 & -2.6 & $\begin{array}{l}\text { 969.5368[M }+\mathrm{Na}]^{+} ; 605.4394[\mathrm{M}-\mathrm{Glc} / \mathrm{Glc}]^{+} \\
587.4312[\mathrm{M}-\mathrm{Glc} / \mathrm{Glc}-\mathrm{OH}]^{+} ; 425.3744[\mathrm{M}- \\
\text { Glc/Glc }-\mathrm{Glc}]^{+} ; 407.3661[\mathrm{M}-\mathrm{Glc} / \mathrm{Glc}-\mathrm{OH}- \\
\mathrm{Glc}^{+}\end{array}$ & $\begin{array}{l}\text { Chikusetsusaponin } \\
\text { FK }_{1}\end{array}$ & $\mathrm{CG}, \mathrm{MCG}_{12}, \mathrm{MCG}_{20}$ & [40] \\
\hline 91 & 12.18 & $\mathrm{C}_{47} \mathrm{H}_{80} \mathrm{O}_{17}$ & 916.5398 & 916.5396 & 0.2 & $\begin{array}{l}961.5380\left[\mathrm{M}+\mathrm{HCOO}^{-} ; 783.4870\left[\mathrm{M}-\mathrm{Xyl}^{-}\right.\right. \\
621.4388[\mathrm{M}-(\mathrm{Xyl} / \mathrm{glc})]^{-}\end{array}$ & $\begin{array}{l}\text { Notoginsenoside } \\
\text { Fe }\end{array}$ & $\mathrm{CG}, \mathrm{MCG}_{12}, \mathrm{MCG}_{20}$ & $\mathrm{~s}$ \\
\hline 92 & 12.39 & $\mathrm{C}_{50} \mathrm{H}_{84} \mathrm{O}_{19}$ & 988.5565 & 988.5607 & -4.1 & $\begin{array}{l}\text { 1011.5458[M }+\mathrm{Na}]^{+} ; 831.4819\left[\mathrm{M}-\mathrm{Glc}^{+}\right. \\
425.3763\left[\mathrm{M}-\mathrm{Glc} / \mathrm{Glc}(\text { ace })-\mathrm{Glc}^{+}\right.\end{array}$ & Quinquenoside III & $\mathrm{CG}, \mathrm{MCG}_{12}, \mathrm{MCG}_{20}$ & [51] \\
\hline 93 & 12.45 & $\mathrm{C}_{47} \mathrm{H}_{80} \mathrm{O}_{17}$ & 916.5376 & 916.5396 & -2.1 & $\begin{array}{l}939.5268[\mathrm{M}+\mathrm{Na}]^{+} ; 789.4754[\mathrm{M}-2 \times \mathrm{OH}- \\
\left.\mathrm{CH}_{6} \mathrm{O}_{3}\right]^{+}\end{array}$ & $\begin{array}{l}\text { Vinaginsenoside } \\
\mathrm{R}_{16}\end{array}$ & $\mathrm{CG}, \mathrm{MCG}_{12}, \mathrm{MCG}_{20}$ & [40] \\
\hline 94 & 12.59 & $\mathrm{C}_{47} \mathrm{H}_{80} \mathrm{O}_{17}$ & 916.5361 & 916.5396 & -3.7 & $\begin{array}{l}939.5253[\mathrm{M}+\mathrm{Na}]^{+} ; 407.3672[\mathrm{M}-\mathrm{Glc}-(\mathrm{Glc} / \mathrm{Xyl}) \\
-\mathrm{OH}]^{+}\end{array}$ & Gypenoside IX & $\mathrm{CG}, \mathrm{MCG}_{12}, \mathrm{MCG}_{20}$ & [52] \\
\hline 95 & 12.91 & $\mathrm{C}_{50} \mathrm{H}_{84} \mathrm{O}_{19}$ & 988.5569 & 988.5607 & -3.8 & $1011.5461[\mathrm{M}+\mathrm{Na}]^{+} ; 789.4784[\mathrm{M}-\mathrm{Glc}-2 \times \mathrm{OH}]^{+}$ & $\begin{array}{l}\text { Quinquenoside III } \\
\text { isomer }\end{array}$ & $\mathrm{CG}, \mathrm{MCG}_{12}, \mathrm{MCG}_{20}$ & [51] \\
\hline 96 & 13.29 & $\mathrm{C}_{52} \mathrm{H}_{86} \mathrm{O}_{19}$ & 1014.5753 & 1014.5763 & -1.0 & $\begin{array}{l}1037.5645[\mathrm{M}+\mathrm{Na}]^{+} ; 857.5032\left[\mathrm{M}-\mathrm{C}_{4} \mathrm{H}_{8} \mathrm{O}_{4}-2 \times\right. \\
\mathrm{OH}]^{+} ; 393.1376[\mathrm{Glc} / \mathrm{Glc}(\text { ace })-\mathrm{OH}]^{+}\end{array}$ & Quinquenoside I & $\mathrm{CG}, \mathrm{MCG}_{12}, \mathrm{MCG}_{20}$ & [53] \\
\hline 97 & 13.34 & $\mathrm{C}_{42} \mathrm{H}_{72} \mathrm{O}_{13}$ & 784.4984 & 784.4973 & 1.4 & $\begin{array}{l}\text { 829.4966[M + } \mathrm{HCOO}^{-} ; 621.4373\left[\mathrm{M}-\mathrm{Glc}^{-}\right. \\
\left.\text {161.0437[Glc }-\mathrm{H}-\mathrm{H}_{2} \mathrm{O}\right]^{-}\end{array}$ & Ginsenoside $\mathrm{F}_{2}$ & $\mathrm{CG}, \mathrm{MCG}_{12}, \mathrm{MCG}_{20}$ & $\mathrm{~s}$ \\
\hline 98 & 13.55 & $\mathrm{C}_{42} \mathrm{H}_{72} \mathrm{O}_{13}$ & 784.4977 & 784.4973 & 0.5 & $\begin{array}{l}807.4869\left[\mathrm{M}+\mathrm{Na}^{+} ; 605.4402\left[\mathrm{M}-\mathrm{Glc}^{+} ;\right.\right. \\
587.4286[\mathrm{M}-\mathrm{Glc}-\mathrm{OH}]^{+} ; 425.3765[\mathrm{M}-\mathrm{Glc} / \mathrm{Glc} \\
-\mathrm{OH}]^{+} ; 407.3659[\mathrm{M}-\mathrm{Glc} / \mathrm{Glc}-2 \times \mathrm{OH}]^{+}\end{array}$ & $\begin{array}{l}\text { 20(R)-Ginsenoside } \\
\operatorname{Rg}_{3}\end{array}$ & $\mathrm{CG}, \mathrm{MCG}_{12}, \mathrm{MCG}_{20}$ & $\mathrm{~s}$ \\
\hline 99 & 13.57 & $\mathrm{C}_{17} \mathrm{H}_{24} \mathrm{O}_{2}$ & 260.1774 & 260.1776 & -0.8 & $261.1847[\mathrm{M}+\mathrm{H}]^{+}$ & Panaxydol & $\begin{array}{c}\Delta \Delta \text {,\#\# } \mathrm{CG}, \mathrm{MCG}_{12} \\
\mathrm{MCG}_{20}\end{array}$ & [54] \\
\hline 100 & 13.77 & $\mathrm{C}_{42} \mathrm{H}_{66} \mathrm{O}_{14}$ & 794.4464 & 794.4453 & 1.4 & $\begin{array}{l}\text { 793.4391[M }-\mathrm{H}]^{-} ; 731.4375\left[\mathrm{M}-\mathrm{CO}_{2}-\mathrm{OH}\right]^{-} \\
613.3746\left[\mathrm{M}-\mathrm{Glc}^{-}\right.\end{array}$ & $\begin{array}{l}\text { Chikusetsusaponin } \\
\text { Iva }\end{array}$ & $\mathrm{CG}, \mathrm{MCG}_{12}, \mathrm{MCG}_{20}$ & [51] \\
\hline 101 & 14.02 & $\mathrm{C}_{52} \mathrm{H}_{86} \mathrm{O}_{19}$ & 1014.5750 & 1014.5763 & -1.3 & $\begin{array}{l}1037.5642[\mathrm{M}+\mathrm{Na}]^{+} ; 789.4732[\mathrm{M}-\mathrm{Glc}- \\
\left.\mathrm{C}_{2} \mathrm{H}_{4} \mathrm{O}_{2}\right]^{+}\end{array}$ & $\begin{array}{c}\text { Isomer of } \\
\text { Quinquenoside I }\end{array}$ & $\mathrm{CG}, \mathrm{MCG}_{12}, \mathrm{MCG}_{20}$ & [51] \\
\hline 102 & 14.38 & $\mathrm{C}_{17} \mathrm{H}_{26} \mathrm{O}_{3}$ & 278.1879 & 278.1882 & -1.1 & $279.1952[\mathrm{M}+\mathrm{H}]^{+}$ & Panaxtriol & $\mathrm{CG}, \mathrm{MCG}_{12}, \mathrm{MCG}_{20}$ & [55] \\
\hline 103 & 14.46 & $\mathrm{C}_{42} \mathrm{H}_{72} \mathrm{O}_{13}$ & 784.4970 & 784.4973 & -0.3 & $\begin{array}{l}\text { 829.4966[M + } \mathrm{HCOO}^{-} ; 621.4373\left[\mathrm{M}-\mathrm{Glc}^{-}\right. \\
\left.\text {407.3672[M }-\mathrm{Glc}^{-} / \mathrm{Glc}-2 \times \mathrm{OH}\right]^{+}\end{array}$ & $\begin{array}{l}\text { 20(S)-Ginsenoside } \\
\operatorname{Rg}_{3}\end{array}$ & $\mathrm{CG}, \mathrm{MCG}_{12}, \mathrm{MCG}_{20}$ & $\mathrm{~s}$ \\
\hline 104 & 15.05 & $\mathrm{C}_{18} \mathrm{H}_{34} \mathrm{O}_{4}$ & 314.2444 & 314.2457 & -3.8 & $337.2336[\mathrm{M}+\mathrm{Na}]^{+}$ & Dibutyl sebacate & $\mathrm{CG}, \mathrm{MCG}_{12}, \mathrm{MCG}_{20}$ & a \\
\hline 105 & 17.90 & $\mathrm{C}_{16} \mathrm{H}_{22} \mathrm{O}_{4}$ & 278.1516 & 278.1518 & -0.7 & $\begin{array}{l}301.1408[\mathrm{M}+\mathrm{Na}]^{+} ; 149.0230\left[\mathrm{M}-\mathrm{C}_{4} \mathrm{H}_{9}-\right. \\
\left.\mathrm{C}_{4} \mathrm{H}_{9} \mathrm{O}\right]^{+}\end{array}$ & $\begin{array}{l}n \text {-Butyl isobutyl } \\
\text { phthalate }\end{array}$ & $\mathrm{CG}, \mathrm{MCG}_{12}, \mathrm{MCG}_{20}$ & a \\
\hline
\end{tabular}


Table 2. Cont

\begin{tabular}{|c|c|c|c|c|c|c|c|c|c|}
\hline No. & $t_{R}(\min )$ & Formula & $\begin{array}{l}\text { Calculated } \\
\text { Mass (Da) }\end{array}$ & $\begin{array}{l}\text { Theoretical } \\
\text { Mass (Da) }\end{array}$ & $\begin{array}{l}\text { Mass Error } \\
\text { (ppm) }\end{array}$ & $\mathrm{MS}^{\mathrm{E}}$ Fragmentation & Identification & Sources & Ref. \\
\hline 106 & 17.93 & $\mathrm{C}_{30} \mathrm{H}_{52} \mathrm{O}_{4}$ & 476.3856 & 476.3866 & -2.2 & $\begin{array}{l}499.3747[\mathrm{M}+\mathrm{Na}]^{+} ; 441.3728[\mathrm{M}-2 \times \mathrm{OH}]^{+} ; \\
423.3590[\mathrm{M}-3 \times \mathrm{OH}]^{+} ; 317.2049\left[\mathrm{M}-2 \times \mathrm{CH}_{3}-\right. \\
\left.\mathrm{C}_{8} \mathrm{H}_{15} \mathrm{O}\right]^{+}\end{array}$ & \multicolumn{2}{|c|}{ 20(R)-ProtopanaxatriolCG, $\mathrm{MCG}_{12}, \mathrm{MCG}_{20}$} & [56] \\
\hline 107 & 17.95 & $\mathrm{C}_{16} \mathrm{H}_{30} \mathrm{O}_{2}$ & 254.2246 & 254.2268 & 8.2 & $277.2161[\mathrm{M}+\mathrm{Na}]^{+}$ & Palmitoleic acid & $\begin{array}{c}\mathrm{CG},{ }^{\Delta, * *} \mathrm{MCG}_{12} \\
\mathrm{MCG}_{20}\end{array}$ & $\mathrm{~s}$ \\
\hline 108 & 18.07 & $\mathrm{C}_{19} \mathrm{H}_{18} \mathrm{O}_{3}$ & 294.1258 & 294.1256 & 0.5 & $317.1150[\mathrm{M}+\mathrm{Na}]^{+}$ & Tashinone IIA & $\mathrm{CG}, \mathrm{MCG}_{12}, \mathrm{MCG}_{20}$ & [57] \\
\hline 109 & 18.08 & $\mathrm{C}_{30} \mathrm{H}_{48} \mathrm{O}_{4}$ & 472.3546 & 472.3553 & -1.4 & $495.3438[\mathrm{M}+\mathrm{Na}]^{+}$ & $\beta$-Amyrone & $\mathrm{CG}, \mathrm{MCG}_{12}, \mathrm{MCG}_{20}$ & [58] \\
\hline 110 & 18.08 & $\mathrm{C}_{6} \mathrm{H}_{6} \mathrm{O}_{3}$ & 126.0331 & 126.0317 & 9.4 & $149.0223[\mathrm{M}+\mathrm{Na}]^{+}$ & Pyrogallol & $\mathrm{CG}, \mathrm{MCG}_{12}, \mathrm{MCG}_{20}$ & a \\
\hline 111 & 18.09 & $\mathrm{C}_{30} \mathrm{H}_{48} \mathrm{O}_{4}$ & 472.3546 & 472.3553 & -1.8 & $495.3438[\mathrm{M}+\mathrm{Na}]^{+}$ & $\begin{array}{l}\text { 24-Hydroxyoleanolic } \\
\text { acid }\end{array}$ & 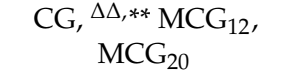 & [59] \\
\hline 112 & 18.09 & $\mathrm{C}_{24} \mathrm{H}_{38} \mathrm{O}_{5}$ & 406.2720 & 406.2719 & 0.3 & $\begin{array}{l}429.2613[\mathrm{M}+\mathrm{Na}]^{+} ; 319.1950\left[\mathrm{M}-\mathrm{CH}_{3}-\mathrm{C}_{4} \mathrm{H}_{7} \mathrm{O}\right]^{+} \\
261.2213\left[\mathrm{M}-2 \times \mathrm{C}_{2} \mathrm{H}_{4} \mathrm{O}_{2}-\mathrm{C}_{2} \mathrm{H}_{3}\right]^{+} ;\end{array}$ & Vitetrifolin & $\mathrm{CG}, \mathrm{MCG}_{12}, \mathrm{MCG}_{20}$ & a \\
\hline 113 & 20.14 & $\mathrm{C}_{31} \mathrm{H}_{46} \mathrm{O}_{2}$ & 450.3535 & 450.3498 & 8.0 & $473.3428[\mathrm{M}+\mathrm{Na}]^{+} ; 430.2889\left[\mathrm{M}-\mathrm{C}_{3} \mathrm{H}_{7}\right]^{+}$ & Vitamin $\mathrm{K}_{1}$ & $\mathrm{CG}, \mathrm{MCG}_{12}, \mathrm{MCG}_{20}$ & [60] \\
\hline 114 & 20.97 & $\mathrm{C}_{18} \mathrm{H}_{30} \mathrm{O}_{2}$ & 278.2224 & 278.2252 & -7.9 & $277.2151[\mathrm{M}-\mathrm{H}]^{-} ; 232.2172[\mathrm{M}-\mathrm{COOH}]^{-}$ & $\alpha$-Linolenic acid & $\begin{array}{c}\Delta \Delta, \# \text { CG, } \mathrm{MCG}_{12} \\
\mathrm{MCG}_{20}\end{array}$ & {$[61]$} \\
\hline 115 & 21.18 & $\mathrm{C}_{21} \mathrm{H}_{38} \mathrm{O}_{4}$ & 354.2758 & 354.2770 & -3.1 & $\begin{array}{l}\text { 377.2650[M + Na }]^{+} \\
265.2525[\mathrm{M}+\mathrm{H}]^{+} ; 149.1320\left[\mathrm{M}-\mathrm{CH}_{2}-\mathrm{C}_{6} \mathrm{H}_{12} \mathrm{O}\right]^{+}\end{array}$ & $\beta$-Monolinolein & $\mathrm{CG}, \mathrm{MCG}_{12}, \mathrm{MCG}_{20}$ & [62] \\
\hline 116 & 22.11 & $\mathrm{C}_{18} \mathrm{H}_{32} \mathrm{O}$ & 264.2452 & 264.2453 & -0.5 & $\begin{array}{l}135.1166\left[\mathrm{M}-\mathrm{CH}_{2}-\mathrm{C}_{7} \mathrm{H}_{13} \mathrm{O}\right]^{+} ; 121.1008[\mathrm{M}- \\
\left.\mathrm{CH}_{2}-\mathrm{C}_{8} \mathrm{H}_{15} \mathrm{O}\right]^{+} ; 109.1010\left[\mathrm{M}-\mathrm{C}_{8} \mathrm{H}_{15} \mathrm{O}-\right. \\
\left.\mathrm{C}_{2} \mathrm{H}_{3}\right]^{+}\end{array}$ & (Z)-9,17-Octadecadien & $\mathrm{aCG}, \mathrm{MCG}_{12}, \mathrm{MCG}_{20}$ & [63] \\
\hline 117 & 22.49 & $\mathrm{C}_{18} \mathrm{H}_{32} \mathrm{O}_{2}$ & 280.2386 & 280.2402 & -5.9 & $279.2313[\mathrm{M}-\mathrm{H}]^{-} ; 234.2325[\mathrm{M}-\mathrm{COOH}]^{-}$ & Linoleic acid & $\begin{array}{c}\Delta \Delta, \# \text { CG, } \mathrm{MCG}_{12} \\
\mathrm{MCG}_{20}\end{array}$ & $\mathrm{~s}$ \\
\hline 118 & 23.85 & $\mathrm{C}_{14} \mathrm{H}_{20} \mathrm{O}_{2}$ & 220.1478 & 220.1463 & 5.6 & $265.1460\left[\mathrm{M}+\mathrm{HCOO}^{-}\right.$ & $\begin{array}{l}\text { Thymyl } \\
\text { isobutyrate }\end{array}$ & $\mathrm{CG}, \mathrm{MCG}_{12}, \mathrm{MCG}_{20}$ & [64] \\
\hline 119 & 24.25 & $\mathrm{C}_{18} \mathrm{H}_{34} \mathrm{O}_{2}$ & 282.2541 & 282.2559 & -6.3 & $281.2468[\mathrm{M}-\mathrm{H}]^{-} ; 236.2481[\mathrm{M}-\mathrm{COOH}]^{-}$ & $\begin{array}{l}\text { 9-Octadecenoic } \\
\text { acid }\end{array}$ & $\begin{array}{c}\Delta \Delta, \# \# C^{C G}, \mathrm{MCG}_{12} \\
\mathrm{MCG}_{20}\end{array}$ & a \\
\hline 120 & 24.40 & $\mathrm{C}_{36} \mathrm{H}_{62} \mathrm{O}_{8}$ & 622.4454 & 622.4445 & 1.6 & $\begin{array}{l}\text { 623.4527[M + H }]^{+} ; 316.2842[\mathrm{M}-\mathrm{OH}-\mathrm{Glc}- \\
\left.\mathrm{C}_{8} \mathrm{H}_{14}\right]^{+}\end{array}$ & Compound $\mathrm{K}$ & $\mathrm{CG}, \mathrm{MCG}_{12}, \mathrm{MCG}_{20}$ & {$[40]$} \\
\hline 121 & 24.89 & $\mathrm{C}_{40} \mathrm{H}_{56} \mathrm{O}_{4}$ & 600.4219 & 600.4179 & 6.7 & $601.4292[\mathrm{M}+\mathrm{H}]^{+} ; 557.4021\left[\mathrm{M}-\mathrm{C}_{2} \mathrm{H}_{4}\right]^{+}$ & Violaxanthin & $\mathrm{CG}, \mathrm{MCG}_{12}, \mathrm{MCG}_{20}$ & [65] \\
\hline 122 & 25.31 & $\mathrm{C}_{20} \mathrm{H}_{38} \mathrm{O}_{2}$ & 310.2862 & 310.2872 & -3.2 & $311.2935[\mathrm{M}+\mathrm{H}]^{+} ; 277.1995\left[\mathrm{M}-\mathrm{C}_{6} \mathrm{H}_{13}\right]^{+}$ & Ethyloleate & $\mathrm{CG}, \mathrm{MCG}_{12}, \mathrm{MCG}_{20}$ & a \\
\hline 123 & 25.35 & $\mathrm{C}_{40} \mathrm{H}_{56} \mathrm{O}_{4}$ & 600.4212 & 600.4179 & 5.6 & $\begin{array}{l}\text { 601.4285[M + H }]^{+} ; 497.3800\left[\mathrm{M}-\mathrm{OH}-\mathrm{C}_{4} \mathrm{H}_{8} \mathrm{O}_{2}\right]^{+} \\
413.2653[\mathrm{M}+\mathrm{Na}]^{+} ; 301.1406\left[\mathrm{M}-3 \times \mathrm{C}_{2} \mathrm{H}_{5}\right]^{+}\end{array}$ & Neoxanthine & $\mathrm{CG}, \mathrm{MCG}_{12}, \mathrm{MCG}_{20}$ & [65] \\
\hline 124 & 26.38 & $\mathrm{C}_{24} \mathrm{H}_{38} \mathrm{O}_{4}$ & 390.2758 & 390.2770 & -2.8 & $\begin{array}{l}189.0153\left[\mathrm{M}-\mathrm{C}_{2} \mathrm{H}_{5}-\mathrm{C}_{4} \mathrm{H}_{9}-\mathrm{C}_{8} \mathrm{H}_{17}\right]^{+} \\
167.0327\left[\mathrm{M}-2 \times \mathrm{C}_{2} \mathrm{H}_{17}\right]^{+}\end{array}$ & $\begin{array}{l}\text { Bis(2-ethylhexyl) } \\
\text { phthalate }\end{array}$ & $\mathrm{CG}, \mathrm{MCG}_{12}, \mathrm{MCG}_{20}$ & a \\
\hline 125 & 28.01 & $\mathrm{C}_{30} \mathrm{H}_{46} \mathrm{O}_{5}$ & 486.3334 & 486.3345 & -2.2 & $509.3226[\mathrm{M}+\mathrm{Na}]^{+}$ & Quillaic acid & $\mathrm{CG}, \mathrm{MCG}_{12}, \mathrm{MCG}_{20}$ & $\mathrm{~s}$ \\
\hline 126 & 29.04 & $\mathrm{C}_{5} \mathrm{H}_{8} \mathrm{O}_{2}$ & 100.0512 & 100.0524 & -10.0 & $123.0404[\mathrm{M}+\mathrm{Na}]^{+}$ & Pentanedial & $\mathrm{CG}, \mathrm{MCG}_{12}, \mathrm{MCG}_{20}$ & [66] \\
\hline
\end{tabular}




\section{Ginsenosides}

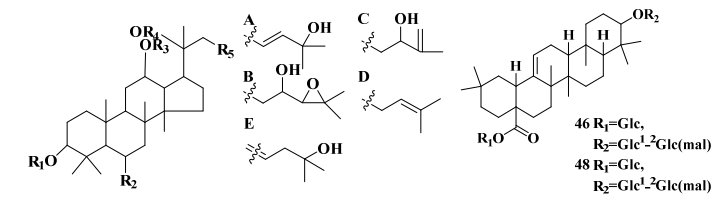

$19 \mathbf{R}_{1}=\mathbf{R}_{3}=\mathbf{H}, \mathbf{R}_{2}=\mathbf{G l e}^{2}-\mathbf{R h a}^{-} \mathbf{R}_{4}=\mathrm{Glc}, \mathbf{R}_{5}=\mathbf{A}$ $25 R_{1}=R_{3}=H, R_{2}=G c^{2}-1$ Glc(Mal), $R_{4}=G i c, R_{5}=D$ $26 \mathbf{R}_{1}=\mathbf{R}_{3}=\mathbf{R}_{4}=\mathbf{H}, \mathbf{R}_{2}=\mathrm{Glc}^{2}-\mathrm{R}^{-1} \mathbf{R h a}, \mathbf{R}_{5}=\mathrm{B}$

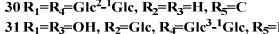

$31 \mathbf{R}_{1}=\mathbf{R}_{3}=\mathrm{OH}, \mathbf{R}_{2}=\mathbf{G l c}, \mathbf{R}_{7}=\mathbf{G l k}{ }^{3}{ }^{-1} \mathbf{G l c}, \mathbf{R}_{5}=\mathbf{D}$

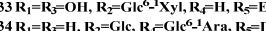

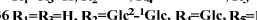

$37 \mathbf{R}_{1}=\mathbf{H}, \mathbf{R}_{2}=\mathbf{G l c}^{2} \mathbf{1}_{-1} \mathbf{M a n}^{2}, \mathbf{R}_{3}=\mathbf{R}_{f}=\mathbf{H}, \mathbf{R}_{5}=\mathbf{C}$

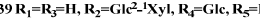

$41 \mathbf{R}_{1}=\mathbf{R}_{3}=\mathbf{H}, \mathbf{R}_{2}=\mathbf{G l c}^{4}-\mathbf{G l c}, \mathbf{R}_{4}=\mathbf{G l c}, \mathbf{R}_{5}=$

$42 R_{1}=R_{3}=-H, R_{2}=G l l^{3}-G l c, R_{4}=G l c, R_{5}-1$

等

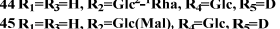

$47 R_{1}=R_{3}=H, R_{2}=R h a(M a l)^{2}-{ }_{-1} G\left|c, R_{1}=G\right| c, R_{5}=D$

$49 R_{1}=R_{3}=H, R_{2}=X y l(m a l), R_{7}=G l c, R_{2}=D$

$50 \mathrm{R}_{1}=\mathrm{R}_{3}=\mathrm{H}, \mathrm{R}_{2}=\mathrm{Glc}(\mathrm{Mal}), \mathrm{R}_{4}=\mathrm{Glc}, \mathrm{R}_{5}=$

$51 \mathbf{R}_{1}=\mathbf{R}_{3}=\mathbf{R}_{4}=\mathbf{H}, \mathbf{R}_{2}=\mathbf{G l c}^{2}-\mathbf{X y l}, \mathbf{R}_{5}=\mathbf{D}$

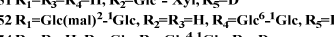

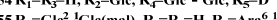

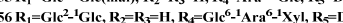

$57 \mathbf{R}_{1}=\mathbf{R}_{3}=\mathbf{R}_{f}=\mathbf{H}, \mathbf{R}_{2}=$ Glc $^{2}-I_{\text {Glc, }} \mathbf{R}_{5}=D$

$59 \mathbf{R}_{1}=\mathbf{R}_{3}=\mathrm{H}, \mathbf{R}_{2}=\mathrm{OH}, \mathbf{R}_{7}-\mathrm{Glc}^{2}-{ }^{-1} \mathrm{Xyl}, \mathbf{R}_{5}=\mathrm{D}$

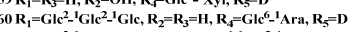

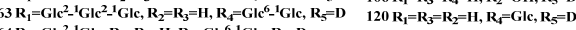

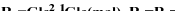

$69 R_{1}=R_{3}=H, R_{2}=O H, R_{4}=G_{l i c}{ }^{6-1} A r a, R_{5}=1$ $74 \mathrm{R}_{1}=\mathrm{Gl}(\mathrm{mal})^{2}-\mathrm{Glc}, \mathrm{R}_{2}=\mathrm{R}_{3}=\mathrm{H}, \mathbf{R}_{4}=\mathrm{Glc}^{6}-\mathrm{Ara}(\mathrm{f}), \mathrm{R}_{5}=1$

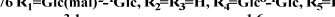
$79 \mathbf{R}_{1}=\mathbf{R}_{3}=\mathbf{R}_{4}=\mathrm{H}, \mathbf{R}_{2}=\mathbf{G l c}, \mathbf{R}_{5}=\mathbf{D}$

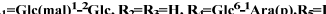

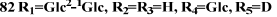

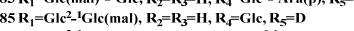

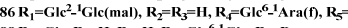

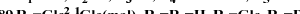
$91 \mathrm{R}_{1}=\mathrm{Glc}, \mathrm{R}_{2}=\mathrm{H}, \mathrm{R}_{3}=\mathrm{H}, \mathrm{R}_{4}=\mathrm{Glc}^{6}{ }^{-1} \mathrm{Xly}, \mathrm{R}_{5}=\mathrm{D}$

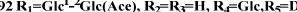
$93 R_{1}=G_{1 c}-R_{\text {Rha }} R_{2}=R_{3}=H, R_{4}=G l c, R_{5}=D$ $94 R_{1}=G l c, R_{3}=R_{2}=H, R_{4}=G l c-X y l, R_{5}=D$

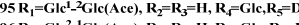

Ace), $\mathbf{R}_{2}=\mathbf{R}_{3}=\mathbf{H}, \mathbf{R}_{\mathbf{F}}=\mathbf{G l c}, \mathbf{R}_{\mathbf{5}}=\mathrm{D}$

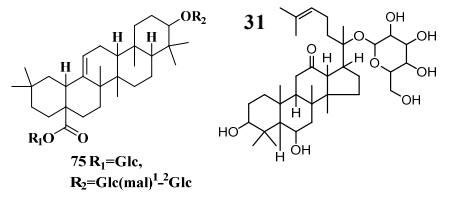

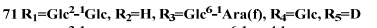

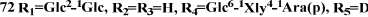

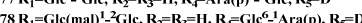
$(p), R_{5}=D$ $\mathbf{R}_{1}=\mathbf{G l c}, \mathbf{R}_{2}=\mathbf{R}_{3}=\mathbf{H}, \mathbf{R}_{7}=\mathbf{G l c}, \mathbf{R}_{5}=\mathbf{D}$

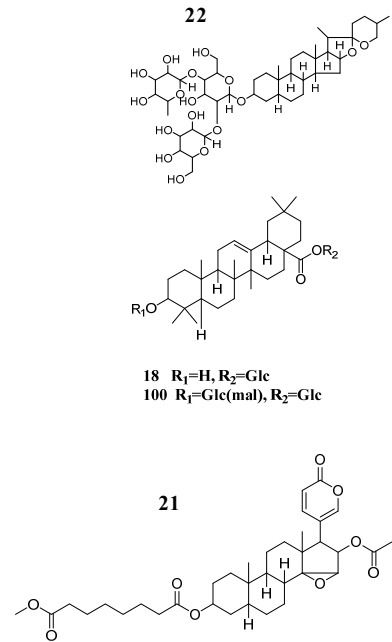

Aldehydes and ketones
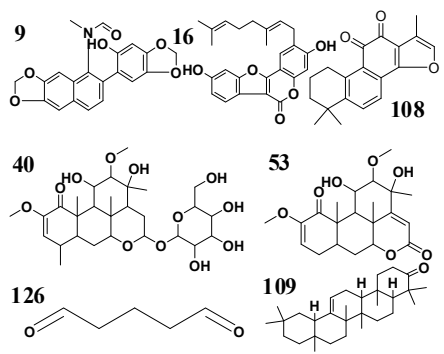

Phenylpropanoids

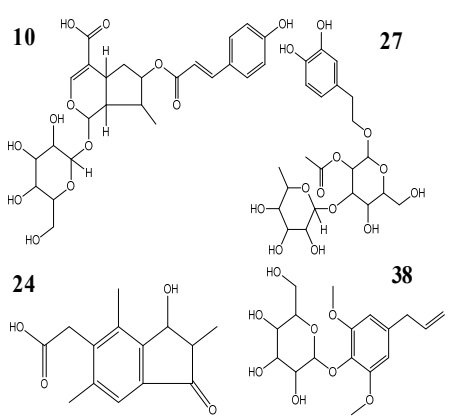

Alcohols and phenols

Amino acids
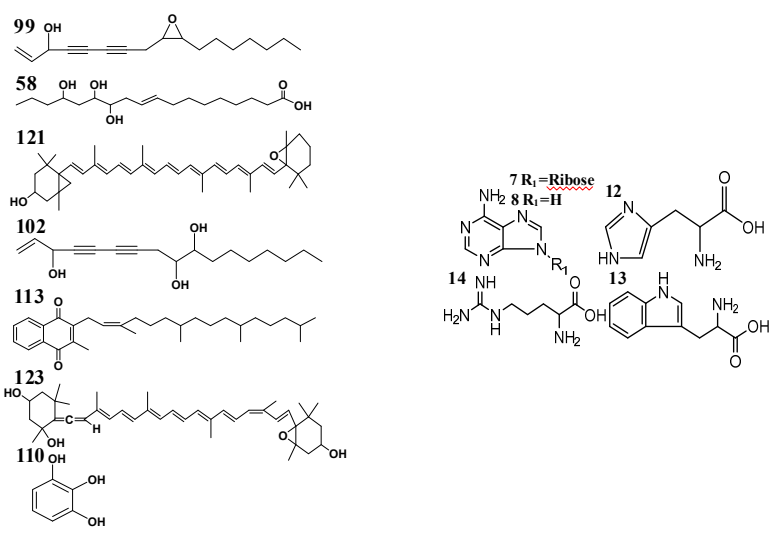

Others

Flavonoids
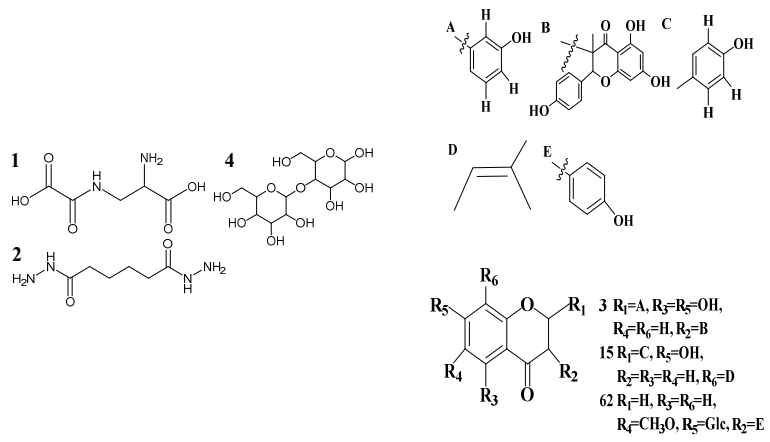

Figure 2. Cont. 
Organic acids and organic acid esters
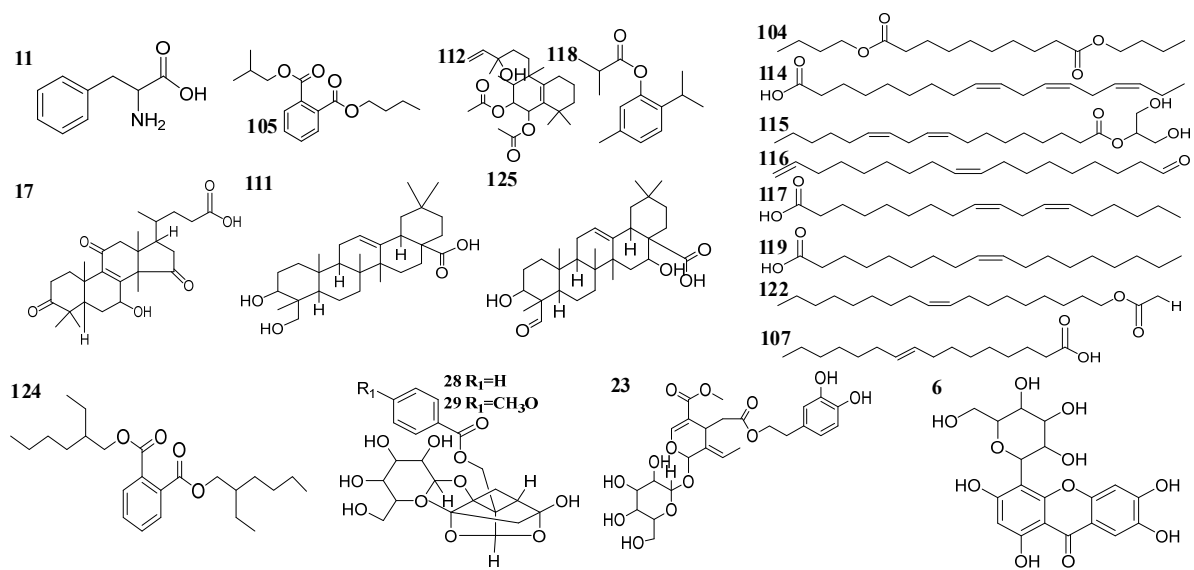

Figure 2. Chemical structures of compounds identified in MCG and CG.

For the isomers, they could be compared with the retention time of the standards or distinguished by the characteristic MS fragmentation patterns reported in literature. Taking compounds $\mathbf{8 2}$ and $\mathbf{8 8}$ as example, both of them had the same protonated ion $[\mathrm{M}+\mathrm{HCOO}]^{-}$at $m / z 991.5464$ and 991.5476 . In a result, one of them was identified as ginsenoside Rd due to the same retention time, and the other one was tentatively identified as gypenoside XVII because it was matched with the characteristic MS fragmentation pattern of gypenoside XVII reported in the literature [31].

\subsection{Biomarker Discovery for Distinguishing MCG and CG}

The MSE data of CG and MCG samples were statistically analyzed via PCA and OPLS-DA. As seen in PCA 2D plots (Figure 3), there was no obvious difference among of 4-6-year-old CG samples, but the $M C G_{20}$ years, $M_{12}$ years and $C_{4-6}$ years groups were obviously separated, indicating that these three groups could be differentiated. With the aim of distinguishing MCG from CG, or $\mathrm{MCG}_{20}$ years from $\mathrm{MCG}_{12 \text { years, }}$ OPLS-DA plot, permutation test, and S-plot, VIP values were obtained to understand which variables were responsible for the separation (Figures 4-6). The variables showing VIP $>1$ and $p<0.05$ (in $t$-test) were considered as potential biomarkers. The robust known biomarkers enabling the differentiation between CG and MCG were discovered and marked in S-plots. In order to systematically evaluate the biomarkers, heatmaps (Figure 7) were generated from these biomarkers. The hierarchical clustering heatmaps, intuitively visualizing the differential levels of potential biomarkers concentration in different ginseng groups, are shown in Figure 7. The larger contents were represented by red squares and smaller values by green squares.
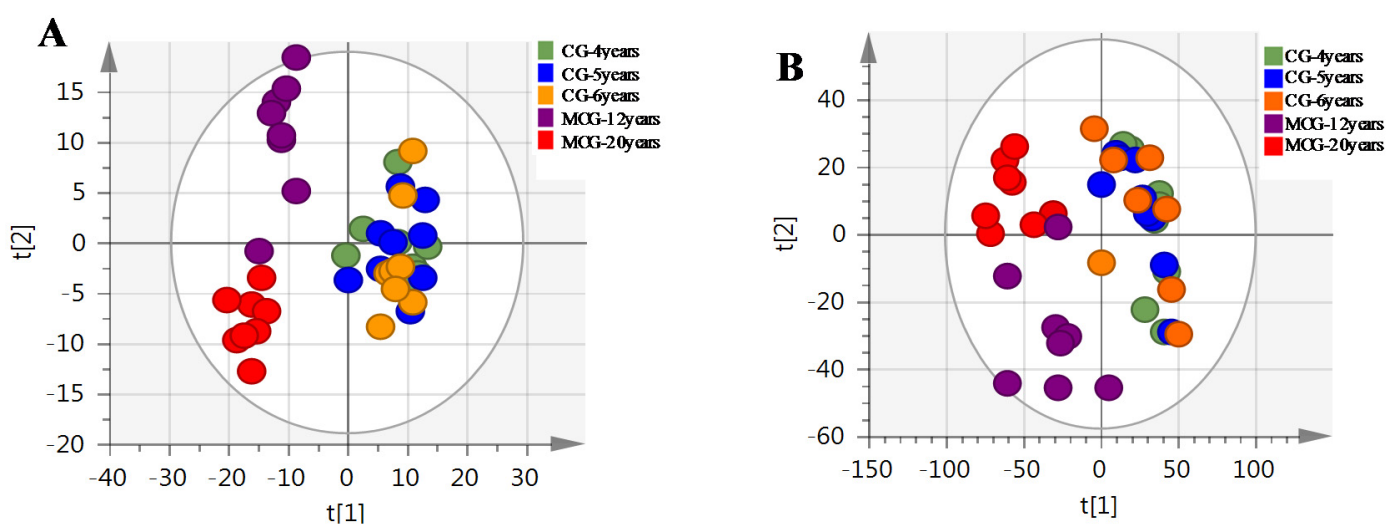

Figure 3. The PCA of CG and MCG in positive mode (A) and negative mode (B). 

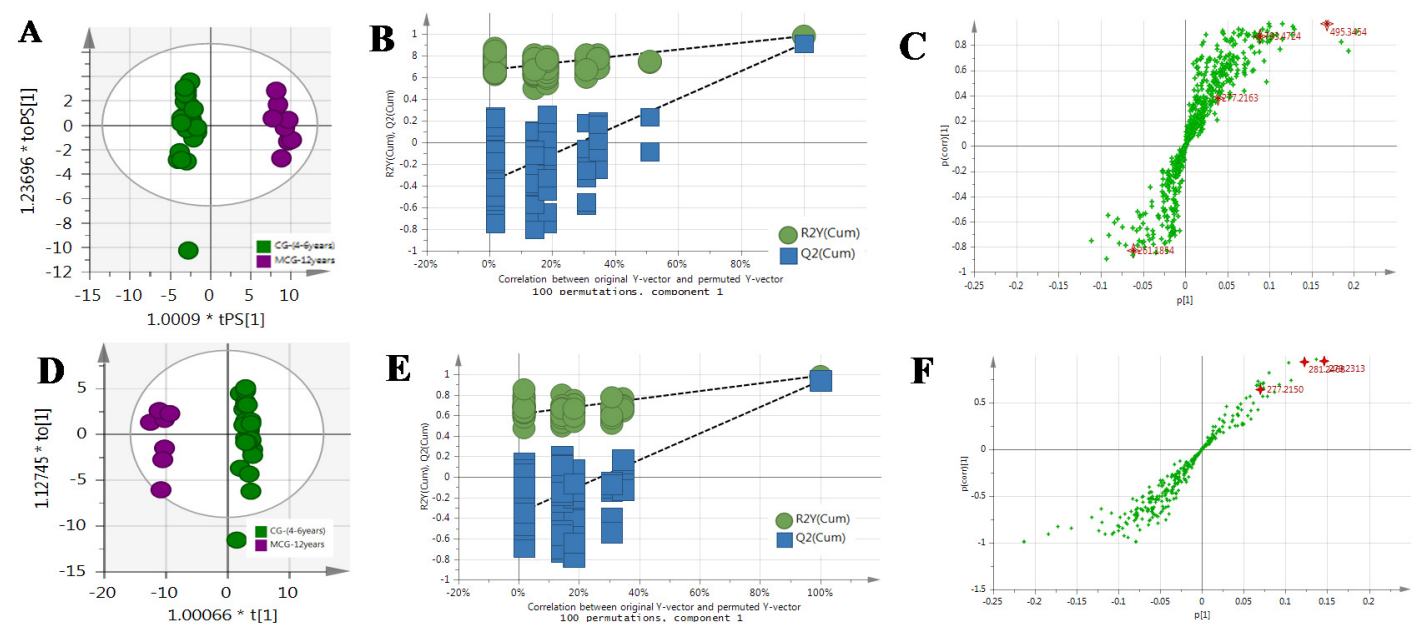

Figure 4. The OPLS-DA/Permutation test/S-Plot of $\mathrm{CG}_{4-6}$ years and $\mathrm{MCG}_{12}$ years in positive mode $(\mathrm{A} / \mathrm{B} / \mathrm{C})$ and negative mode $(\mathrm{D} / \mathrm{E} / \mathrm{F})$.
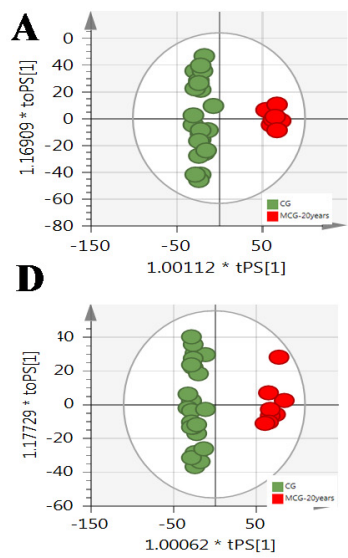
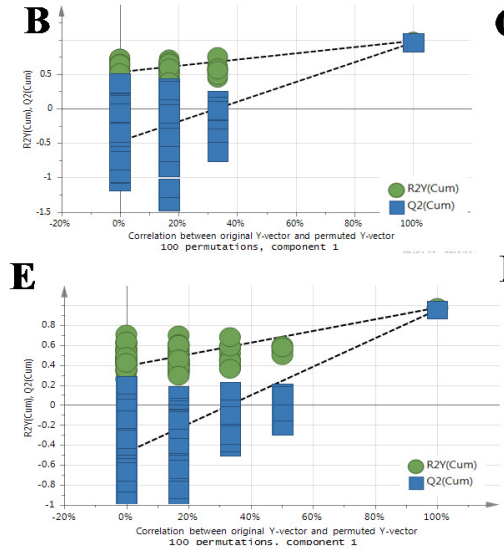

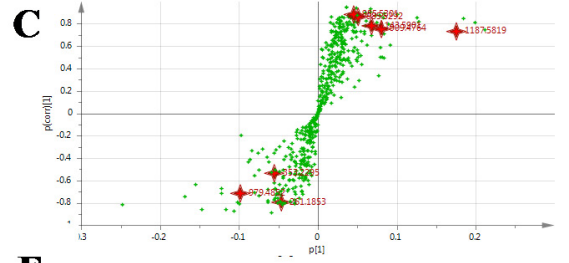

F

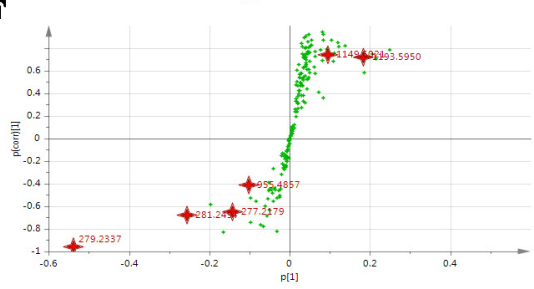

Figure 5. The OPLS-DA/Permutation test/S-Plot of $\mathrm{CG}_{4-6}$ years and $\mathrm{MCG}_{20}$ years. in positive mode $(\mathrm{A} / \mathrm{B} / \mathrm{C})$ and negative mode $(\mathrm{D} / \mathrm{E} / \mathrm{F})$.

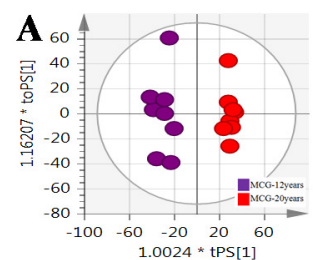

B
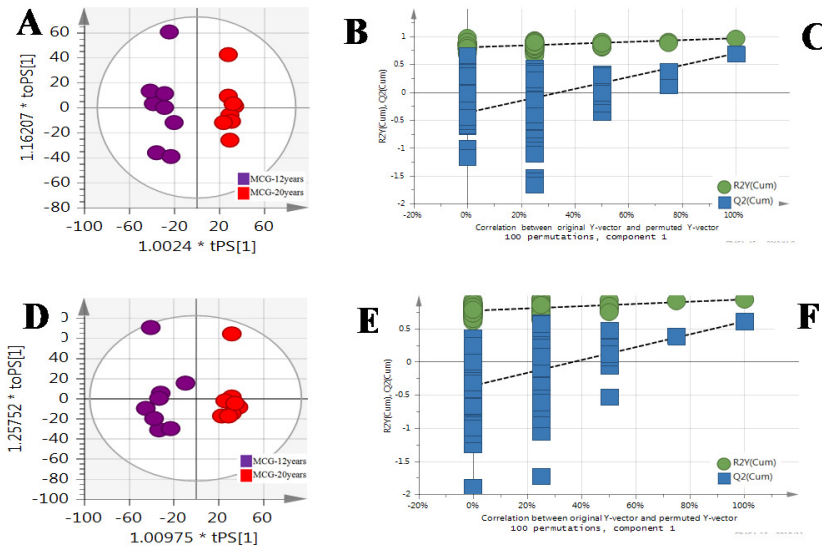

C
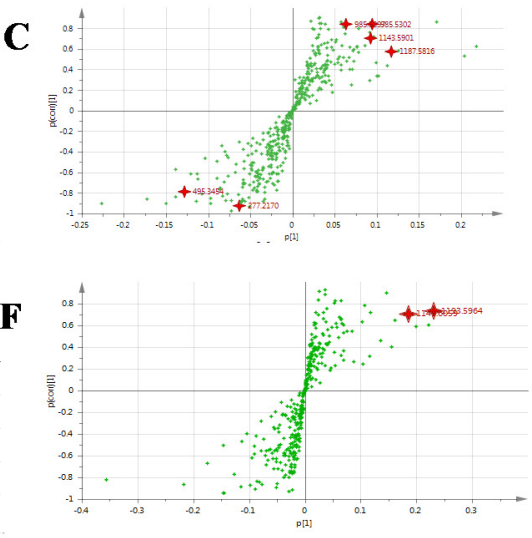

Figure 6. The OPLS-DA/Permutation test/S-Plot of $\mathrm{MCG}_{12}$ years and $\mathrm{MCG}_{20}$ years in positive mode $(\mathbf{A} / \mathbf{B} / \mathbf{C})$ and negative mode $(\mathrm{D} / \mathrm{E} / \mathrm{F})$. 


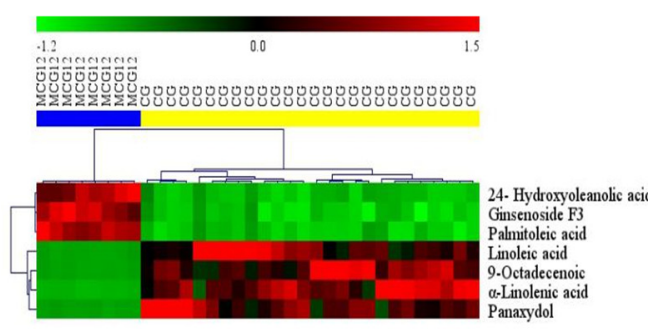

$\mathrm{MCG}_{2}$ vs CG

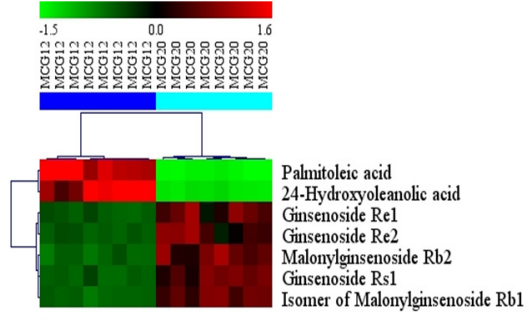

$\mathrm{MCG}_{2}$ vs $\mathrm{MCG}_{20}$

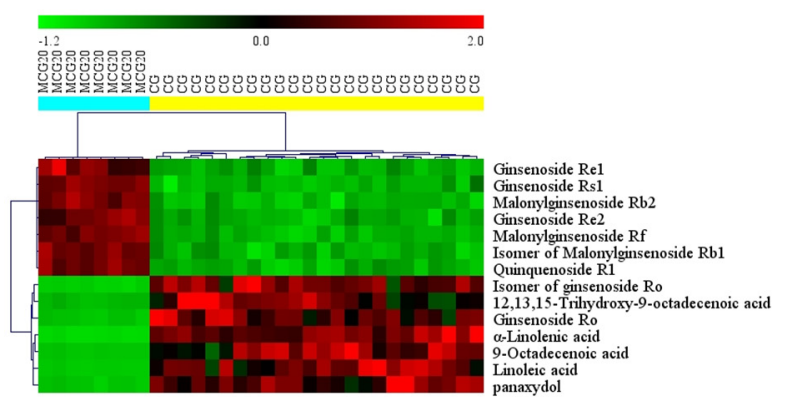

$\mathrm{MCG}_{20} v \mathrm{vSG}$

Figure 7. The heatmaps visualizing the intensities of potential biomarkers.

Between the $\mathrm{CG}_{4-6 \text { years }}$ and $\mathrm{MCG}_{12 \text { years }}$ groups, the contents of 24-hydroxyoleanolic acid, ginsenoside $\mathrm{F}_{3}$ and palmitoleic acid in $\mathrm{MCG}_{12}$ samples were significantly higher. While, the contents of $\alpha$-linolenic acid, 9-octadecenoic acid, linoleic acid and panaxydol in all the CG samples were significantly higher.

Between the $\mathrm{CG}_{4-6}$ years and $M \mathrm{MG}_{20}$ years groups, the contents of ginsenoside $\operatorname{Re}_{1},-\operatorname{Re}_{2},-\mathrm{Rs}_{1}$, malonylginsenoside $R b_{2}$, $-R f$, isomer of malonylginsenoside- $R b_{1}$ and quinquenoside $R_{1}$ in the samples of $\mathrm{MCG}_{20}$ years were higher. On the contrary, the contents of ginsenoside Ro and the isomer of ginsenoside Ro, 12,13,15-trihydroxy-9-octadecenoic acid, linoleic acid, 9-octadecenoic acid, $\alpha$-linolenic acid, panaxydol were rather higher in CG samples.

Between the $\mathrm{MCG}_{12}$ years and $\mathrm{MCG}_{20}$ years groups, the contents of palmitoleic acid and 24-hydroxyoleanolic acid in $\mathrm{MCG}_{12}$ years samples were significantly high, while the contents of ginsenoside $R_{1},-R_{1}$, malonylginsenoside $R b_{2},-R_{2}$ and isomer of malonylginsenoside $R_{1}$ were rather higher in $\mathrm{MCG}_{20}$ years samples.

Overall, on one hand, the contents of $\alpha$-linolenic acid, linoleic acid, 9-octadecenoic acid and panaxydol in CG samples were significantly higher than those in all MCG samples. On the other hand, ginsenoside $R_{1},-\mathrm{Re}_{2},-\mathrm{Rs}_{1}$, malonylginsenoside $\mathrm{Rb}_{2}$ and isomer of malonylginsenoside $R b_{1}$ in $\mathrm{MCG}_{20}$ years samples were really higher than those both in $\mathrm{MCG}_{12}$ years and in all of $C G$ samples, but there is no significant difference between $\mathrm{MCG}_{12}$ years and $\mathrm{CG}_{4-6}$ years samples. The summary with variable identity, VIP and $p$ value were shown in Table 3. 
Table 3. The summary table with variable identity, VIP and $\mathrm{p}$ value.

\begin{tabular}{|c|c|c|c|c|}
\hline Groups fo & mparison & Marker' Name & VIP Value & $p$ Value \\
\hline \multirow{7}{*}{$\begin{array}{l}\mathrm{CG}_{4-6} \text { years vs. } \\
\mathrm{MCG}_{12} \text { years }\end{array}$} & \multirow{4}{*}{$\mathrm{CG}_{4-6}$ years } & $\alpha$-linolenic acid & 1.23 & $<0.001$ \\
\hline & & 9-octadecenoic acid & 2.17 & $<0.001$ \\
\hline & & linoleic acid & 2.57 & $<0.001$ \\
\hline & & panaxydol & 1.49 & $<0.001$ \\
\hline & \multirow{3}{*}{$\mathrm{MCG}_{12}$ years } & 24-hydroxyoleanolic acid & 4.13 & $<0.001$ \\
\hline & & ginsenoside F3 & 2.15 & $<0.001$ \\
\hline & & palmitoleic acid & 1.54 & 0.037 \\
\hline \multirow{14}{*}{$\begin{array}{l}\mathrm{CG}_{4-6} \text { years vs. } \\
\mathrm{MCG}_{20 \text { years }}\end{array}$} & \multirow{7}{*}{$\mathrm{MCG}_{20 \text { years }}$} & ginsenoside Re1 & 1.60 & $<0.001$ \\
\hline & & ginsenoside $\operatorname{Re} 2$ & 1.75 & $<0.001$ \\
\hline & & ginsenoside Rs1 & 1.59 & $<0.001$ \\
\hline & & malonylginsenoside $\mathrm{Rb} 2$ & 4.10 & $<0.001$ \\
\hline & & ginsenoside $\mathrm{Rf}$ & 1.83 & $<0.001$ \\
\hline & & isomer of malonylginsenoside $\mathrm{Rb} 1$ & 2.30 & $<0.001$ \\
\hline & & quinquenoside R1 & 1.21 & $<0.001$ \\
\hline & \multirow{7}{*}{$\mathrm{CG}_{4-6}$ years } & ginsenoside Ro & 1.39 & 0.017 \\
\hline & & isomer of ginsenoside Ro & 2.31 & 0.022 \\
\hline & & 12,13,15-trihydroxy-9-octadecenoic acid & 1.25 & 0.003 \\
\hline & & linoleic acid & 7.08 & $<0.001$ \\
\hline & & 9-octadecenoic acid & 3.45 & $<0.001$ \\
\hline & & $\alpha$-linolenic acid & 1.86 & $<0.001$ \\
\hline & & panaxydol & 1.12 & $<0.001$ \\
\hline \multirow{7}{*}{$\begin{array}{c}\mathrm{MCG}_{12 \text { years }} \mathrm{vs} . \\
\mathrm{MCG}_{20 \text { years }}\end{array}$} & \multirow{2}{*}{$\mathrm{MCG}_{12}$ years } & palmitoleic acid & 2.07 & $<0.001$ \\
\hline & & 24-hydroxyoleanolic acid & 3.26 & $<0.001$ \\
\hline & \multirow{5}{*}{$\mathrm{MCG}_{20 \text { years }}$} & ginsenoside Re1 & 1.16 & 0.002 \\
\hline & & ginsenoside Rs1 & 1.89 & 0.024 \\
\hline & & malonylginsenoside $\mathrm{Rb} 2$ & 2.76 & 0.026 \\
\hline & & ginsenoside $\operatorname{Re} 2$ & 1.60 & $<0.001$ \\
\hline & & isomer of malonylginsenoside-Rb1 & 3.87 & $<0.001$ \\
\hline
\end{tabular}

\section{Discussion}

Although MCG and CG both belong to Panax ginseng, their chemical ingredients and pharmacological activities are different due to their significantly different growth environment $[3,67]$. As we all know, MCG has been regarded as a replacement of wild ginseng. Recently, the UPLCQTOF-MS/MS-based approach has been developed to distinguish MCG (grown for 15 years) and CG (grown for 4-7 years) [6]. As a result, 40 ginsenosides in both MCG and CG were unambiguously identified and tentatively assigned, and the potential chemical markers identifying different ginseng products were characterised [6]. Additionally, the study on 6-18-year-old Mountain Cultivated Ginseng Leaves (MGL) samples showed that the MGL were obviously divided into three main groups according to different age brackets (6 10, 11 13 and 14 18 years) [7]. Although the sample of the study was the leaf of MCG, it could be indirectly speculated that the MCG roots with different cultivation ages are also different. In order to further systematically compare the similarities and differences at the chemical level between different ages of ginseng, especially to compare the younger or the older MCG, 4, 5, 6-year-old CG and 12, 20-year-old MCG were chosen as the analytical samples in the present study.

Firstly, based on UNIFI platform, intelligent and automatic workflows, the screening analysis of metabolites in different cultivation ages of ginseng were rapidly performed. As a result, a total of 126 compounds were characterized from $C_{4-6}$ years, $M_{C G} 12$ years and $M_{C G} C_{20}$ years samples. Among of them, ginsenosides were the main ingredients. Both CG and MCG had the similar chemical composition, but the components were variously distributed in CG and MCG samples at different contents. That means in CG and MCG, the secondary metabolites had the features of structural diversity and the different content patterns. As far as we know, this is the first time that the comprehensive 
screening analysis of $\mathrm{MCG}_{12}$ years and $\mathrm{MCG}_{20}$ years samples by using UPLC-QTOF-MS ${ }^{\mathrm{E}}$ combined with UNIFI platform. It could provide the scientific data for clarifying the chemical composition of MCG.

Secondly, the combination of LC-MS based metabolomic profiling with multivariate statistical analysis method was used to profile the $C G, M C G_{12}$ years and $M_{C} G_{20}$ years samples. A total of 17 potential age-dependent markers enabling differentiation among the CG and MCG samples were discovered. (1) There were four robust markers including $\alpha$-linolenic acid, 9-octadecenoic acid, linoleic acid and panaxydol being the characteristic components for CG samples, that distinguished them from both $\mathrm{MCG}_{12}$ years and $\mathrm{MCG}_{20}$ years samples. The results showed that $C G$ samples contained more non-ginsenosides. Both linoleic acid and $\alpha$-linolenic acid, the main products of the acetate-malonate pathway, are two essential fatty acids necessary for health. Linoleic acid is used in the biosynthesis of arachidonic acid and thus some prostaglandins, leukotrienes, and thromboxane [68,69]. Panaxydol, one of the C17 polyacetylenic compounds, originates from acetyl-CoA/malonyl-CoA via fatty acids with crepenynate as the intermediate [70]. It is considered a potential antitumor agent due to its significant anticancer activity [71]. (2) In CG samples, there were three other characteristic components such as ginsenoside Ro, the isomer of ginsenoside Ro, and 12,13,15-trihydroxy-9-octadecenoic acid, that could be used to differentiate them from $\mathrm{MCG}_{20}$ years samples. From this, we could draw a conclusion that pentacyclic triterpenoids decreased significantly in older MCG samples. (3) Five robust biomarkers including ginsenoside $\mathrm{Re}_{1},-\mathrm{Re}_{2}$, $-\mathrm{Rs}_{1}$, malonylginsenoside $\mathrm{Rb}_{2}$ and isomer of malonylginsenoside $R b_{1}$ were found to enable differentiation of $M C_{20}$ years from $C G$ and $M C G_{12}$ years samples. These five compounds might be used for rapid identification of $\mathrm{MCG}_{20}$ years samples. A proposed biosynthetic pathway of ginsenosides is as follows: with the action of squalene epoxidase, squalene was converted to 2,3-oxidosqualene. Dammaranes can be synthesized by dammarenediol synthase, and oleananes by $\beta$-amyrin synthase [72]. Ginsenosides were found to have both antimicrobial and antifungal properties and the molecules are naturally bitter-tasting, discouraging insects and other animals from consuming the plant, so ginsenosides likely serve as mechanisms for plant defense [73,74]. (4) In $M C G_{20}$ years samples, another two markers, ginsenoside $\mathrm{Rf}$ and quinquenoside $\mathrm{R}_{1}$, were discovered that distinguished them from all CG samples. (5) In MCG $_{12}$ years samples, 24-hydroxyoleanolic acid and palmitoleic acid were the two robust markers for distinguished from both $C G$ and $\mathrm{MCG}_{20}$ years samples. These two compounds might be used for rapid identification of $\mathrm{MCG}_{12}$ years samples. Palmitoleic acid is biosynthesized from palmitic acid by the action of the enzyme stearoyl-CoA desaturase-1, a key enzyme in fatty acid metabolism [75]. (6) Ginsenoside $\mathrm{F}_{3}$ was another marker for $\mathrm{MCG}_{12}$ years samples that differentiated them from CG samples. However, there are still some unresolved issues. For example, as shown in BPI chromatograms, though 126 compounds were identified, there are still some unidentified components. there are still some unidentified components. Further research should be carried out based on the formula of these unknown compounds.

\section{Conclusions}

By combining the UPLC-Q/TOF-MS ${ }^{\mathrm{E}}$ and UNIFI platform, 126 chemical components with various structural types, such as triterpenoids, flavonoids, organic acids and organic acid esters, etc., were characterized or tentatively identified from $C_{4-6}$ years, $\mathrm{MCG}_{12}$ years and $\mathrm{MCG}_{20}$ years samples for the first time. All the CG and MCG samples had the similar chemical composition, but there were significant differences in the content of each component. Further nontarget metabolomic analysis combined with multivariate statistical analysis showed that $C_{4-6}$ years, $M_{C G}$ years and $M C_{20}$ years samples were obviously divided into three different groups. A total of 17 potential age-dependent markers enabling differentiation among the CG and MCG samples were discovered. Among of these markers, four robust markers, including $\alpha$-linolenic acid, 9-octadecenoic acid, linoleic acid and panaxydol, could be the characteristic components for differentiation of CG from all other MCG samples. Five robust markers including ginsenoside $\operatorname{Re}_{1},-\mathrm{Re}_{2},-\mathrm{Rs}_{1}$, malonylginsenoside $\mathrm{Rb}_{2}$ and isomer of malonylginsenoside $\mathrm{Rb}_{1}$ were found to enable differentiate $\mathrm{MCG}_{20}$ years samples from all other samples, while 24-hydroxyoleanolic acid and palmitoleic acid were the robust markers for 
distinguishing $\mathrm{MCG}_{12}$ years samples from all the $C G$ samples and $\mathrm{MCG}_{20}$ years samples. The proposed approach could be applied to directly distinguish MCG root ages, which is an important criterion for evaluating the quality of MCG. The results will provide the data for the deficient study on the chemical constituents of MCG and provide reference for the quantitative determination in the quality control criterion of MCG.

Author Contributions: The individual contributions of authors are specified as following: Data curation, InvestigationWriting-original draft, H.Z.; Methodology, Software, H.L.; Formal analysis, Writing-original draft, J.T.; Components identification, Writing editing, C.W.; Conceptualization, Methodology, H.W.; Investigation, F.W.; Data curation, Q.D.; Writing-review and editing, Y.L.; Funding acquisition, P.L.; Supervision, J.L.

Funding: This research was supported by the Jilin Province Science and Technology Department for Science and Technology Development Project of Jilin Province [No. 20160307008YY].

Conflicts of Interest: The authors declare that they have no conflict of interest concerning this article.

\section{References}

1. Liu, D.; Li, Y.G.; Xu, H.; Sun, S.Q.; Wang, Z.T. Differentiation of the root of Cultivated Ginseng, Mountain Cultivated Ginseng and Mountain Wild Ginseng using FT-IR and two-dimensional corre- lation IR spectroscopy. J. Mol. Struct. 2008, 883, 228-235. [CrossRef]

2. Jung, C.H.; Seog, H.M.; Choi, I.W.; Cho, H.Y. Antioxidant activities of cultivated and wild Korean ginseng leaves. Food Chem. 2005, 92, 535-540. [CrossRef]

3. Kim, S.J.; Shin, S.S.; Seo, B.I.; Jee, S.Y. Effect of mountain grown ginseng radix, mountain cultivated ginseng radix, and cultivated ginseng radix on apoptosis of HL-60 cells. J. Herb. 2004, 19, 19-41.

4. Pan, H.Y.; Qu, Y.; Zhang, J.K.; Kang, T.G.; Dou, D.Q. Antioxidant activity of ginseng cultivated under mountainous forest with different growing years. J. Ginseng Res. 2013, 37, 355-360. [CrossRef] [PubMed]

5. Xiao, D.; Yue, H.; Xiu, Y.; Sun, X.L.; Wang, Y.B.; Liu, S.Y. Accumulation characteristics and correlation analysis of five ginsenosides with different cultivation ages from different regions. J. Ginseng Res. 2015, 39, 338-344. [CrossRef] [PubMed]

6. Xu, X.F.; Cheng, X.L.; Lin, Q.H.; Li, S.S.; Jia, Z.; Han, T.; Lin, R.C.; Wang, D.; Wei, F.; Li, X.R. Identification of mountain-cultivated ginseng and cultivated ginseng using UPLC/oa- TOF MSE with a multivariate statistical sample-profiling strategy. J. Ginseng Res. 2016, 40, 344-350. [CrossRef] [PubMed]

7. Chang, X.W.; Zhang, J.J.; Li, D.K.; Zou, D.Z.; Zhang, Y.L.; Wang, J.C.; Hu, B.; Ju, A.C.; Ye, Z.L. Nontargeted metabolomics approach for the differentiation of cultivation ages of mountain cultivated ginseng leaves using UHPLC/QTOF-MS. J. Pharm. Biomed. Anal. 2017, 141, 108-122. [CrossRef] [PubMed]

8. Corthout, J.; Naessens, T.; Apers, S.; Vlietinck, A.J. Quantitative determination of ginsenosides from Panax ginseng roots and ginseng preparations by thin layer chromatography-densitometry. J. Pharm. Biomed. Anal. 1999, 21, 187-192. [CrossRef]

9. Wei, S.; Wang, Y.T.; Li, J.; Zhang, H.Q.; Ding, L. Investigation of ginsenosides in different parts and ages of Panax ginseng. Food Chem. 2007, 102, 664-668.

10. Yong, E.C.; Yong, S.K.; Yi, M.J.; Park, W.G.; Yi, J.S.; Chun, S.R.; Han, S.S.; Lee, S.J. Physiological and chemical characteristics of field-and mountain-cultivated ginseng roots. J. Plant Biol. 2007, 50, 198-205.

11. Wu, W.; Jiao, C.X.; Li, H.; Ma, Y.; Jiao, L.L.; Liu, S.Y. LC-MS based metabolic and metabonomic studies of Panax ginseng. Phytochem. Anal. 2018, 29, 331-340. [CrossRef] [PubMed]

12. Qiu, S.; Yang, W.Z.; Yao, C.L.; Qiu, Z.D.; Shi, X.J.; Zhang, J.X.; Hou, J.J.; Wang, Q.R.; Wu, W.Y.; Guo, D.A. Nontargeted metabolomic analysis and "commercial-homophyletic" comparison-induced biomarkers verification for the systematic chemical differentiation of five different parts of Panax ginseng. J. Chromatogr. A 2016, 33, 78-87. [CrossRef] [PubMed]

13. Pace, R.; Martinelli, E.M.; Sardone, N.; Combarieu, E.D.E. Metabolomic evaluation of ginsenosides distribution in Panax genus (Panax ginseng and Panax quinquefolius) using multivariate statistical analysis. Fitoterapia 2015, 101, 80-91. [CrossRef] [PubMed]

14. Wang, H.P.; Liu, Y.; Chang, C.; Xiao, H.B. Screening Specific Biomarkers of Herbs Using a Metabolomics Approach: A Case Study of Panax ginseng. Sci. Rep. 2017, 7, 1-9. [CrossRef] [PubMed] 
15. Chang, X.W.; Li, D.K.; Wang, T.; Wu, Y.C.; Zhao, Y.; Zhou, D.Z.; Zhang, T.; Ye, Z.L. Application of metabolomics approach to study of different parts of Mountain Cultivated Ginseng using UHPLC-QTOF/MS. Acta Pharm. Sin. B 2016, 51, 1609-1615.

16. In, G.; Seo, H.K.; Park, H.W.; Jang, K.H. A Metabolomic Approach for the Discrimination of Red Ginseng Root Parts and Targeted Validation. Molecules 2017, 22, 471. [CrossRef] [PubMed]

17. Shin, J.S.; Park, H.W.; In, G.; Seo, H.K.; Won, T.H.; Jang, K.H.; Cho, B.G.; Han, C.K.; Shin, J.H. Metabolomic approach for discrimination of four- and six-year-old red ginseng (Panax ginseng) using UPLC-QToF-MS. Chem. Pharm. Bull. 2016, 64, 1298-1303. [CrossRef]

18. Kim, N.; Kim, K.; Choi, B.Y.; Lee, D.H.; Shin, Y.S.; Bang, K.H.; Cha, S.W.; Lee, J.W.; Choi, H.K.; Jang, D.S.; et al. Metabolomic approach for age discrimination of Panax ginseng using UPLC-Q-TOF MS. J. Agric. Food Chem. 2011, 59, 10435-10441. [CrossRef]

19. Zhang, F.X.; Li, M.; Qiao, L.R.; Yao, Z.H.; Li, C.; Shen, X.Y.; Wang, Y.; Yu, K.; Yao, X.S.; Dai, Y. Rapid characterization of Ziziphi Spinosae Semen by UPLC/Qtof MS with novel informatics platform and its application in evaluation of two seeds from Ziziphus species. J. Pharm. Biomed. Anal. 2016, 122, 59-80. [CrossRef]

20. Wang, C.Z.; Zhang, N.Q.; Wang, Z.Z.; Qi, Z.; Zhu, H.L.; Zheng, B.Z.; Li, P.Y.; Liu, J.P. Nontargeted Metabolomic Analysis of Four Different Parts of Platycodon grandiflorum Grown in Northeast China. Molecules 2017, 22, 1280. [CrossRef]

21. Wang, Y.R.; Wang, C.Z.; Lin, H.Q. Discovery of the Potential Biomarkers for Discrimination between Hedyotis diffusa and Hedyotis corymbosa by UPLC-QTOF/MS Metabolome Analysis. Molecules 2018, 23, 1525. [CrossRef] [PubMed]

22. National Pharmacopoeia Commission. Pharmacopoeia of the People's Republic of China, 2015 Version; China Medical Science and Technology Press: Beijing, China, 2015.

23. Koh, L.H.; Lau, A.J.; Chan, C.Y. Hydrophilic interaction liquid chromatography with tandem mass spectrometry for the determination of underivatized dencichine ( $\beta$ - $N$-oxalyl-L- $\alpha, \beta$-diaminopropionic acid) in Panax medicinal plant species. Rapid Commun. Mass Spectrom. 2005, 19, 1237-1244. [CrossRef] [PubMed]

24. Tüting, W.; Adden, R.; Mischnick, P. Fragmentation pattern of regioselectively O-methylated maltooligosaccharides in electrospray ionisation-mass spectrometry/collision induced dissociation. Int. J. Mass Spectrom. 2004, 232, 107-115. [CrossRef]

25. Wang, H.; Sun, H.; Zhang, A.; Li, Y.; Wang, L.; Shi, H.; Li Dizou, X.; Wang, X. Rapid identification and comparative analysis of the chemical constituents and metabolites of Phellodendri amurensis cortex and Zhibai dihuang pill by ultra-performance liquid chromatography with quadrupole TOF-MS. J. Sep. Sci. 2013, 36, 3874-3882. [PubMed]

26. Chen, M.L.; Chang, W.Q.; Zhou, J.L.; Yin, Y.H.; Xia, W.R.; Liu, J.R.; Liu, L.F.; Xin, G.Z. Comparison of three officinal species of Callicarpa based on a biochemome profiling strategy with UHPLC-IT-MS and chemometrics analysis. J. Pharm. Biomed. Anal. 2017, 145, 666-674. [CrossRef]

27. Fuhrer, T.; Heer, D.; Begemann, B.; Zamboni, N. High-throughput, accurate mass metabolome profiling of cellular extracts by flow injection-time-of-flight mass spectrometry. Anal. Chem. 2011, 83, 7074-7080. [CrossRef]

28. Song, W.; Qiao, X.; Chen, K.; Wang, Y.; Ji, S.; Feng, J.; Li, K.; Lin, Y.; Ye, M. Biosynthesis-Based Quantitative Analysis of 151 Secondary Metabolites of Licorice to Differentiate Medicinal Glycyrrhiza Species and Their Hybrids. Anal. Chem. 2017, 89, 3146-3153. [CrossRef]

29. Li, Y.; Li, C.; Yu, J.; Gao, Y.; Zhao, Y.; Xue, D.; Zhang, G.Q.; Chai, Y.F.; Ke, Y.; Zhang, H. Rapid separation and characterization of comprehensive ingredients in Yangxinshi tablet and rat plasma by ultrahigh-performance liquid chromatography-quadrupole time-of-flight mass spectrometry. J. Liq. Chromatogr. Relat. Technol. 2017, 59, 339-354. [CrossRef]

30. Wang, H.Y.; Hua, H.Y.; Liu, X.Y.; Liu, J.H.; Yu, B.Y. In vitro biotransformation of red ginseng extract by human intestinal microflora: Metabolites identification and metabolic profile elucidation using LC-Q-TOF/MS. J. Pharm. Bio. Anal. 2014, 98, 296-306. [CrossRef]

31. Yang, W.Z.; Ye, M.; Qiao, X.; Liu, C.F.; Miao, W.J.; Bo, T.; Tao, H.Y.; Guo, D.A. A strategy for efficient discovery of new natural compounds by integrating orthogonal column chromatography and liquid chromatography/mass spectrometry analysis: Its application in Panax ginseng, Panax quinquefolium and Panax notoginseng to characterize 437 potential new ginsenosides. Anal. Chim. Acta 2012, 739, 56-66. 
32. Jaiswal, Y.; Liang, Z.; Ho, A.; Chen, H.; Zhao, Z. A Comparative Tissue-specific Metabolite Analysis and Determination of Protodioscin Content in Asparagus Species used in Traditional Chinese Medicine and Ayurveda by use of Laser Microdissection, UHPLC-QTOF/MS and LC-MS/MS. Phytochem. Anal. Pca. 2014, 25, 514-528. [CrossRef] [PubMed]

33. Li, H.; Yao, W.; Liu, Q.; Xu, J.; Bao, B.; Shsn, M.Q.; Cao, Y.D.; Cheng, F.F.; Ding, A.; Zhang, L. Application of UHPLC-ESI-Q-TOF-MS to Identify Multiple Constituents in Processed Products of the Herbal Medicine Ligustri Lucidi Fructus. Molecules 2017, 22, 689. [CrossRef]

34. Wu, W.; Sun, L.; Zhang, Z.; Guo, Y.Y.; Liu, S.Y. Profiling and multivariate statistical analysis of Panax ginseng based on ultra-high-performance liquid chromatography coupled with quadrupole-time-of-flight mass spectrometry. J. Pharm. Bio. Anal. 2015, 107, 141-150. [CrossRef]

35. Yang, L.; Ying, P.; Wang, M.Y.; Zhou, G.S.; Zhang, Y.L.; Li, X. Rapid screening and identification of the differences between metabolites of Cistanche deserticola, and C. tubulosa, water extract in rats by UPLC-Q-TOF-MS combined pattern recognition analysis. J. Pharm. Bio. Anal. 2016, 131, 364-372.

36. Zhang, Y.; Cheng, Y.; Liu, Z.; Ding, L.Q.; Qiu, T.Y.; Chai, L.W.; Qiu, F.; Wang, Z.Z.; Xiao, W.; Zhao, L.S.; Chen, X.H. Systematic screening and characterization of multiple constituents in Guizhi Fuling capsule and metabolic profiling of bioactive components in rats using ultra-high-performance liquid chromatography/ quadrupole-time-of-flight mass spectrometry. J. Chromatogr. B 2017, 1061, 474-486. [CrossRef]

37. Tang, S.Y.; Liu, S.; Liu, Z.Q.; Song, F.R.; Liu, S.Y. Analysis and Identification of the Chemical Constituents of Ding-Zhi-Xiao-Wan Prescription by HPLC-IT-MSn and HPLC-Q-TOF-MS. Chinese J. Chem. 2015, 33, 451-462. [CrossRef]

38. Chen, L.L.; Qi, J.; Chang, Y.X.; Zhu, D.N.; Yu, B.Y. Identification and determination of the major constituents in Traditional Chinese Medicinal formula Danggui-Shaoyao-San by HPLC-DAD-ESI-MS/MS. J. Pharm. Biomed. Anal. 2009, 50, 127-137. [CrossRef] [PubMed]

39. Chen, Y.J.; Xu, L.; Zhao, Y.C.; Zhao, Z.Z.; Chen, H.B.; Yi, T.; Qin, M.J.; Liang, Z.T. Tissue-specific metabolite profiling and quantitative analysis of ginsenosides in Panax quinquefolium, using laser microdissection and liquid chromatography-quadrupole/time of flight-mass spectrometry. Chem. Cent. J. 2015, 9, 66-72. [CrossRef]

40. Qiu, S.; Yang, W.Z.; Shi, X.J.; Yao, C.L.; Yang, M.; Liu, X.; Jiang, B.H.; Wu, W.Y.; Guo, D.A. A green protocol for efficient discovery of novel natural compounds: Characterization of new ginsenosides from the stems and leaves of Panax ginseng as a case study. Anal. Chim. Acta 2015, 893, 65-76. [CrossRef] [PubMed]

41. Dan, M.; Su, M.M.; Gao, X.F.; Zhao, T.; Zhao, A.H.; Xie, G.X.; Qiu, Y.P.; Zhou, M.M.; Liu, Z.; Jia, W. Metabolite profiling of Panax notoginseng using UPLC-ESI-MS. Phytochemistry 2008, 69, 2237-2244. [CrossRef]

42. Wu, Q.L.; Wang, M.F.; Simon, J.E.; Yu, S.C.; Xiao, P.G.; Ho, C.T. Studies on the Chemical Constituents of Loquat Leaves (Eriobotrya japonica). ACS Sym. 2003, 28, 292-306.

43. Wang, L.L.; Han, L.F.; Yu, H.S.; Sang, M.M.; Liu, E.W.; Zhang, Y.; Fang, S.M.; Wang, T.; Gao, X.M. Analysis of the Constituents in "Zhu She Yong Xue Shuan Tong" by Ultra High Performance Liquid Chromatography with Quadrupole Time-of-Flight Mass Spectrometry Combined with Preparative High Performance Liquid Chromatography. Molecules 2015, 20, 20518-20537. [CrossRef] [PubMed]

44. Li, S.L.; Lai, S.F.; Song, J.Z.; Qiao, C.F.; Liu, X.; Zhou, Y.; Cai, H.; Cai, B.C.; Xu, H.X. Decocting-induced chemical transformations and global quality of Du-Shen-Tang, the decoction of ginseng evaluated by UPLC-Q-TOF-MS/MS based chemical profiling approach. J. Pharm. Biomed. Anal. 2010, 53, 946-957. [CrossRef] [PubMed]

45. Liang, Z.T.; Chen, Y.J.; Liang, X.; Qin, M.J.; Yi, T.; Chen, H.B.; Zhao, Z.Z. Localization of ginsenosides in the rhizome and root of Panax ginseng, by laser microdissection and liquid chromatography-quadrupole/time of flight-mass spectrometry. J. Pharm. Biomed. Anal. 2015, 105, 121-133. [CrossRef]

46. Peng, J.; Dou, S.S.; Liu, L.; Zhang, W.D.; Chen, Z.L.; Xu, R.L.; Ding, J.M. Identification of Multiple Constituents in the TCM-Formula Shexiang Baoxin Pill by LC Coupled with DAD-ESI-MS-MS. Chromatographia 2009, 70, 133-142.

47. Murae, T.; Sugie, A.; Moriyama, Y.; Tsuyuki, T.; Takahashi, T. Mass spectra of the bitter principles fromPicrasma ailanthoides Planchon. J. Mass Spectrom. 1974, 8, 291-301. [CrossRef]

48. Castro, O.N.; Benites, J.L.; Rodilla, J.; Santiago, J.; Simirgiotis, M.; Sepulveda, B.; Areche, C. Metabolomic Analysis of the Lichen Everniopsis trulla Using Ultra High Performance Liquid ChromatographyQuadrupole-Orbitrap Mass Spectrometry (UHPLC-Q-OT-MS). Chromatographia 2017, 80, 1-7. [CrossRef] 
49. Peng, L.; Yu, H.S.; Zhang, L.J.; Song, X.B.; Kang, L.P.; Liu, J.Y.; Zhang, J.; Cao, M.; Yu, K.; Kang, T.J.; Ma, B.P. A rapid method for chemical fingerprint analysis of Pan Panax notoginseng powders by ultra performance liquid chromatography coupled with quadrupole time-of-flight mass spectrometry. Chin. J. Nat. Med. 2015, 13, 471-480.

50. Lai, C.M.; Li, S.P.; Yu, H.; Wan, K.W.; Wang, Y.T. A rapid HPLC-ESI-MS/MS for qualitative and quantitative analysis of saponins in "XUESETONG" injection. J. Pharm. Biomed. Anal. 2006, 40, 669-678. [CrossRef]

51. Sun, J.H.; Chen, P. Differentiation of Panax quinquefolius, grown in the USA and China using LC/MS-based chromatographic fingerprinting and chemometric approaches. Anal. Bioanal. Chem. 2011, 399, 1877-1889. [CrossRef]

52. Wan, J.Y.; Wang, C.Z.; Liu, Z.; Zhang, Q.H.; Musch, M.; Bissonnette, M.; Chang, E.B.; Li, P.; Qi, L.W.; Yuan, C.S. Determination of American ginseng saponins and their metabolites in human plasma, urine and feces samples by liquid chromatography coupled with quadrupole time-of-flight mass spectrometry. J. Chromatogr. B 2016, 62, 1015-1016. [CrossRef] [PubMed]

53. Qi, L.W.; Wang, H.Y.; Zhang, H.; Wang, C.Z.; Li, P.; Yuan, C.S. Diagnostic ion filtering to characterize ginseng saponins by rapid liquid chromatography with time-of-flight mass spectrometry. J. Chromatogr. A 2012, 1230, 93-99. [CrossRef] [PubMed]

54. Xu, G.H.; Choo, S.J.; Ryoo, I.J.; Kim, Y.H.; Paek, K.Y.; Yoo, I.D. Polyacetylenes from the tissue cultured adventitious roots of Panax ginseng C.A. meyer. Nat. Prod. Sci. 2008, 14, 177-181.

55. Yang, H.; Liu, L.; Gao, W.; Liu, K.; Qi, L.W.; Li, P. Direct and comprehensive analysis of ginsenosides and diterpene alkaloids in Shenfu injection by combinatory liquid chromatography-mass spectrometric techniques. J. Pharm. Biomed. Anal. 2014, 92, 13-21. [CrossRef] [PubMed]

56. Yan, L.; Guan, T.Y.; Zhou, Y.Y.; Liu, Y.N.; Xing, L.; Zheng, X.; Dai, C.; Du, P.; Rao, T.; Zhou, L.J.; Yu, X.Y.; Hao, K.; Xie, L.; Wang, G.J. Effect of mobile phase additives on qualitative and quantitative analysis of ginsenosides by liquid chromatography hybrid quadrupole-time of flight mass spectrometry. J. Chromatogr. A 2013, 1297, 29-36.

57. Yang, X.H.; Cheng, X.L.; Qin, B.; Cai, Z.Y.; Cai, X.; Liu, S.; Wang, Q.; Qin, Y. Ultra-high performance liquid chromatography coupled with quadrupole/time of flight mass spectrometry based chemical profiling approach for the holistic quality control of complex Kang-Jing formula preparations. J. Pharm. Biomed. Anal. 2016, 124, 319-336. [CrossRef] [PubMed]

58. Coqueiro, A.; Regasini, L.O.; Leme, G.M.; Polese, L.; Nogueira, C.T.; Cistia, M.D.; Graminha, M.A.S. Leishmanicidal activity of Brosimum glaziovii (Moraceae) and chemical composition of the bioactive fractions by using high-resolution gas chromatography and GC-MS. J. Braz. Chem. Soc. 2014, 25, 1839-1847.

59. Wang, C.Z.; Zhang, N.Q.; Wang, Z.Z.; Qi, Z.; Zheng, B.Z.; Li, P.Y.; Liu, J.P. Rapid characterization of chemical constituents of Platycodon grandiflorum and its adulterant Adenophora stricta by UPLC-QTOF-MS/MS. J. Mass Spectrom. 2017, 52, 643-657. [CrossRef] [PubMed]

60. Huang, B.F.; Zheng, F.F.; Fu, S.S.; Yao, J.H.; Tao, B.H.; Ren, Y.P. UPLC-ESI-MS/MS for determining trans-and cis-vitamin K1 in infant formulas: Method and applications. Eur. Food Res. Tech. 2012, 235, 873-879. [CrossRef]

61. Hurtadofernández, E.; Pacchiarotta, T.; Gómezromero, M. Ultra high performance liquid chromatographytime of flight mass spectrometry for analysis of avocado fruit metabolites: Method evaluation and applicability to the analysis of ripening degrees. J. Chromatogr. A 2011, 1218, 7723-7738. [CrossRef]

62. Doshi, G.M.; Nalawade, V.V.; Mukadam, A.S. Structural elucidation of chemical constituents from Benincasa hispidaseeds andCarissa congestaroots by gas chromatography: Mass spectroscopy. Pharmacognosy Res. 2015, 7, 282-293. [CrossRef] [PubMed]

63. Montserrat, R.A.; Liliana, V.; Stefania, V.; Josep, M.G.; Elvira, L.T.; Susana, B. Characterisation of volatile composition of white salsify (Tragopogon porrifolius L.) by headspace solid-phase microextraction (HS-SPME) and simultaneous distillation-extraction (SDE) coupled to GC-MS. Food Chem. 2011, 129, 557-564.

64. Mathela, C.S.; Singh, K.K.; Gupta, V.K. Synthesis and in vitro antibacterial activity of thymol and carvacrol derivatives. Acta Pol. Pharm. 2010, 67, 375-380. [PubMed]

65. Wang, N.; Manabe, Y.K.; Sugawara, T.; Paul, N.A.; Zhao, J. Identification and biological activities of carotenoids from the freshwater alga Oedogonium intermedium. Food Chem. 2017, 20, 247-255. [CrossRef] [PubMed] 
66. Lee, M.J.; Chung, I.M.; Kim, H.J.; Jung, M.J. High resolution LC-ESI-TOF-mass spectrometry method for fast separation, identification, and quantification of 12 isoflavones in soybeans and soybean products. Food Chem. 2015, 176, 254-262. [CrossRef] [PubMed]

67. Hwang, J.W.; Oh, J.H.; Yoo, H.S.; Lee, Y.W.; Cho, C.K.; Kwon, K.R.; Yoon, J.H.; Park, J.S.; Her, S.; Lee, Z.W. Mountain ginseng extract exhibits anti-lung cancer activity by inhibiting the nuclear translocation of NF- $\mathrm{KB}$. Am. J. Chin. Med. 2012, 40, 187-202. [CrossRef] [PubMed]

68. Carvalho, E.B.T.; Melo, I.L.P.; Mancini-Filho, J. Chemical and physiological aspects of isomers of conjugated fatty acids. Food Sci. Technol. 2010, 30, 295-307. [CrossRef]

69. Harwood, J.L. Recent advances in the biosynthesis of plant fatty acids. Biochim. Biophysica. Acta 1996, 1301, 7-56. [CrossRef]

70. Kim, H.S.; Lim, J.M.; Kim, J.Y.; Park, S.; Sohn, J. Panaxydol, a component of Panax ginseng, induces apoptosis in cancer cells through EGFR activation and ER stress and inhibits tumor growth in mouse models. Int. J. Cancer. 2016, 138, 1432-1441. [CrossRef]

71. Nihat, K.; Elena, O.; Nicholas, S.; Huber, C.; Luis, M.; Bonfill, M. Biosynthesis of Panaxynol and Panaxydol in Panax ginseng. Molecules 2013, 18, 7686-7698.

72. Liang, Y.; Zhao, S. Progress in understanding of ginsenoside biosynthesis. Plant Biol. 2010, 10, 415-421. [CrossRef] [PubMed]

73. Kim, Y.J.; Zhang, D.; Yang, D.C. Biosynthesis and biotechnological production of ginsenosides. Biotechnol. Adv. 2015, 33, 717-735. [CrossRef] [PubMed]

74. Leung, K.W.; Wong, A.S. Pharmacology of ginsenosides: A literature review. Chin. Med. 2010, 5, $20-27$. [CrossRef] [PubMed]

75. Velisek, J.; Cejpek, K. Biosynthesis of food constituents: Lipids. 1. Fatty acids and derived compounds-A review. Czech J. Food Sci. 2006, 24, 193-216. [CrossRef]

Sample Availability: Samples of the compounds are available from the authors.

(C) 2018 by the authors. Licensee MDPI, Basel, Switzerland. This article is an open access article distributed under the terms and conditions of the Creative Commons Attribution (CC BY) license (http://creativecommons.org/licenses/by/4.0/). 